\title{
ALGAS MARINAS BENTÓNICAS DEL LITORAL DE CAMPECHE, MÉXICO
}

\author{
Luz Elena Mateo-Cid ${ }^{1,3}$, A. Catalina Mendoza-González $Z^{1}$ \\ Alejandrina G. Ávila-Ortiz ${ }^{2}$ y Sergio Díaz Martínez ${ }^{2}$ \\ ${ }^{1}$ Instituto Politécnico Nacional, Escuela Nacional de Ciencias Biológicas, \\ Departamento de Botánica, Carpio y Plan de Ayala, \\ Colonia Santo Tomás, 11340 México, D.F., México. \\ ${ }^{2}$ Universidad Nacional Autónoma de México, Facultad de Estudios Superiores \\ Zaragoza, Herbario FEZA, Batalla 5 de Mayo y Fuerte de Loreto, \\ Colonia Ejército de Oriente, 09230 México, D.F., México. \\ ${ }^{3}$ Autor para la correspondencia: 1mateoc@ipn.mx
}

\section{RESUMEN}

Se presentan los resultados obtenidos en un estudio sobre las algas marinas bentónicas en ocho localidades del litoral de Campeche, México. Se determinó la presencia de 211 taxones de algas marinas, siendo el grupo Rhodophyta el más diverso con 102 taxones, sigue en importancia Chlorophyta con 66 especies, seis variedades y cuatro formas, Heterokontophyta con 19 especies y por último Cyanobacteria con 14. La lista florística se acompaña de datos sobre presencia, estacionalidad, reproducción, nivel de marea y referencias De los 211 taxones, 30 son nuevos registros para Campeche, y se cita por primera vez a Centroceras micracanthum y Streblonema invisibile para la costa occidental del Atlántico de México. Por otro lado, se compara la diversidad específica entre la estación climática de lluvias y la de secas durante 2003, 2004, 2006 y 2007. La ficoflora ubicada en Campeche es de afinidad tropical y la diversidad más grande fue encontrada durante la época seca.

Palabras clave: algas marinas, Campeche, distribución, diversidad, México, nuevos registros.

\section{ABSTRACT}

We present results of the study on benthic marine algae in eight localities from the coast of Campeche, Mexico. The results indicate the presence of 211 taxa of marine algae 
with Rhodophyta having the greatest diversity with 102 taxa, followed by Chlorophyta with 66 taxa, six varieties and four forms, Heterokontophyta with 19 species and finally Cyanobacteria with 14 . The floristic list is accompanied by data on presence, seasonality, reproduction, tidal level and references. Of the 211 taxa, 30 are new records for Campeche, with Centroceras micracanthum and Streblonema invisibile being new records for the occidental coast of the Atlantic of Mexico. Species diversity was compared between the rainy and the dry seasons during 2003, 2004, 2006 and 2007. The algal flora of the coast of Campeche is tropical and the greatest diversity was found during the dry season.

Key words: Campeche, distribution, diversity, Mexico, new records, seaweeds.

\section{INTRODUCCIÓN}

Las algas marinas bentónicas son un importante componente del ecosistema marino y de las zonas arrecifales de la Península de Yucatán, ya que son los productores, constructores de los arrecifes y formadores de sustrato. A pesar de la extensión del litoral mexicano del Golfo de México y la abundante literatura ficológica a que ha dado lugar, mencionada por Ortega et al. (2001), resalta lo reducido de las investigaciones en el litoral de Campeche. Entre los estudios realizados en esta región están los de Huerta-Múzquiz y Garza-Barrientos (1966) para la Laguna de Términos; Huerta-Múzquiz et al. (1987) para la Península de Yucatán, en el que se incluyen registros para el litoral de Campeche; Ortega (1995) registró 80 especies recolectadas entre 1964 y 1966 en 16 estaciones de la Laguna de Términos y tres en ambiente marino; y finalmente Callejas-Jiménez et al. (2005) reportaron 51 especies de algas marinas para el litoral continental del estado. En trabajos de naturaleza monográfica, Dreckmann y De Lara-Isassi (2000) citan a Gracilaria caudata de Isla Aguada, y Gurgel et al. (2003) describen a Gracilariopsis cata-luziana para la Laguna de Términos. Asimismo, existen revisiones monográficas sobre las familias Udoteaceae y Sphacelariaceae, así como de la tribu Callithamniae (Mateo-Cid et al., 2003), donde se registran varias especies para Campeche (Mendoza-González et al., 2000, Aguilar-Rosas et al., 2001). En las investigaciones mencionadas anteriormente se encuentran citadas 218 especies de algas marinas para el litoral de Campeche. En este sentido, el presente artículo incorpora nuevos registros y una integración de la composición florística de las algas de la región, así como datos acerca de su variación estacional en las épocas de lluvias y secas. 


\section{MATERIALES Y MÉTODOS}

Área de estudio

Según Carranza-Edwards et al. (1975), el litoral de Campeche pertenece a la cuarta unidad morfotectónica del Golfo de México y Mar Caribe, la que se caracteriza por presentar costas primarias de suspensión subaérea con ríos y presencia de deltas, costas primarias de deposición terrestre con numerosos cañones y sumideros, costas secundarias por deposición marina que originan playas e islas de barrera y costas secundarias por organismos marinos que favorecen la formación de arrecifes coralinos (Pica et al., 1991).

El clima del litoral de Campeche se distribuye de norte a sur desde el semiseco muy cálido y cálido $\left(\mathrm{BS}_{1}\left(\mathrm{~h}^{\prime}\right) \mathrm{w}\left(\mathrm{i}^{\prime}\right)\right.$ y $\mathrm{BS}_{0}\left(\mathrm{~h}^{\prime}\right) \mathrm{w}^{\prime \prime}\left(\mathrm{x}^{\prime}\right)$ hasta el cálido subhúmedo (Aw $\left.{ }_{0}\left(i^{\prime}\right) g w "\right)$ y cálido húmedo (Am)(García, 1983). En el Golfo de México la temperatura está determinada por el influjo de la Corriente del Lazo, que transporta aguas superficiales, tiene forma de herradura y fluye en el sentido de las agujas del reloj, transfiriendo aguas cálidas subtropicales del Mar Caribe a través del Estrecho de Yucatán hacia el Golfo de México.

La capa superficial del agua de mar (hasta los $200 \mathrm{~m}$ de profundidad) presenta temperaturas fluctuantes. En los meses de invierno (diciembre, enero, febrero) se registran valores de 15 a $25^{\circ} \mathrm{C}$, debido a los frentes polares y nortes. Durante el verano (julio, agosto y septiembre) este factor se acentúa hasta alcanzar $28^{\circ} \mathrm{C}$. Tales variaciones se deben principalmente a la influencia de las corrientes de El Lazo y la del Caribe, ambas cálidas, y su alcance de intrusión por el canal de Yucatán oscila durante el año.

En el litoral de Campeche las mareas son diurnas. Las aguas típicas del Golfo de México tienen una salinidad de 36.5 psu, producto de la mezcla de los giros anticiclónicos con la masa de agua subtropical subsuperficial, que tiene una salinidad de 36.6 a una profundidad de 70 m (De la Lanza Espino y Gómez Rojas, 2004).

Trabajo de campo y gabinete

Este trabajo incluyó tres fases: la primera de ellas fue la recolección de material ficológico en ocho localidades del estado de Campeche durante 2003, 2004, 2006 y 2007, mediante cinco muestreos. Los ejemplares se obtuvieron en diferentes sustratos que requieren las algas marinas para su fijación y desarrollo, tales como rocas, guijarros, arena y praderas de pastos marinos, principalmente de Thalassia testudinum Banks ex König. Se realizaron dos muestreos en la estación climática de lluvias (octubre 2004, junio 2006) y tres en la de secas (diciembre 2003, noviembre 2006, abril 2007). 
Las plantas fueron recolectadas a mano con ayuda de espátulas y navajas de campo en el nivel intermareal y solo en Isla Aguada en el submareal por medio de buceo libre hasta una profundidad de seis metros. Las recolectas se realizaron en segmentos de las playas de aproximadamente $500 \mathrm{~m}$ de extensión.

Localidades de muestreo. En el Cuadro 1 se indican los sitios de muestreo con coordenadas geográficas, la profundidad y la fecha de colecta. La ubicación se muestra en la Fig. 1. Las características de cada localidad son las siguientes:

1. Puerto Real: playa con pendiente suave y oleaje moderado, posee una zona rocosa siendo en su mayoría arenosa con praderas de pastos marinos.

2. Isla Aguada: localidad predominantemente arenosa, con oleaje moderado.

3. Sabancuy: playa arenosa con rocas y pequeñas escolleras.

4. $29 \mathrm{~km}$ al NE de Sabancuy: zona con planicies rocosas cubiertas por limo, y pequeños agregados de pastos marinos.

5. Punta Xen: extensa playa arenosa con prominentes agregados rocosos, en algunos puntos el sustrato es limoso y tiene escasas praderas de pastos marinos.

6. Champotón: playa de tipo areno-limoso con grandes extensiones de pastos marinos, además está fuertemente influenciada por actividades pesqueras y de turismo.

7. Playa Bonita: playa preponderantemente arenosa con agregados rocosos y praderas de pastos marinos.

8. Escolleras Ciudad de Campeche: se caracteriza por presentar playas arenosas, rocosas y escolleras con pequeñas praderas de Thalassia testudinum. El lugar también se encuentra influenciado por actividades de turismo, además de que presenta contaminación por descargas de desechos domésticos.

Las muestras se etiquetaron y se fijaron en una solución de formaldehido a $4 \%$ en agua de mar. El material fue incorporado a la sección ficológica del herbario de la Escuela Nacional de Ciencias Biológicas (ENCB) y del herbario de la Facultad de Estudios Superiores-Zaragoza (FEZA). Para la observación de las algas se utilizó un microscopio binocular Olympus CX31RBSF, realizándose cortes de los talos con navajas de doble filo para observar estructuras reproductivas y vegetativas. Para la descalcificación de los representantes de la familia Corallinaceae se utilizó $\mathrm{HNO}_{3}$ 0.6 M. La determinación del material ficológico se llevó a cabo utilizando las claves y descripciones referidas en los trabajos de Taylor (1960), Schneider y Searles (1991), Littler y Littler (2000), Dawes y Mathieson (2008), Cho et al. (2008) y Martín-Les- 
Cuadro 1. Ubicación de las localidades de muestreo, profundidad y fechas de colecta.

\begin{tabular}{|c|c|c|c|}
\hline Localidad & Coordenadas & Profundidad de colecta & Fecha de colecta \\
\hline 1. Puerto Real & $\begin{array}{l}18^{\circ} 46^{\prime} 44.9^{\prime \prime} \mathrm{N} \\
91^{\circ} 31^{\prime} 51.6^{\prime \prime} \mathrm{W}\end{array}$ & $\begin{array}{l}0-2 \text { metros } \\
\text { profundidad }\end{array}$ & $\begin{array}{c}\text { octubre } 2004, \text { junio } \\
\text { y noviembre } 2006, \\
\text { abril } 2007\end{array}$ \\
\hline 2. Isla Aguada & $\begin{array}{l}18^{\circ} 50^{\prime} 44.7^{\prime \prime} \mathrm{N} \\
91^{\circ} 26^{\prime} 9.3^{\prime \prime} \mathrm{W}\end{array}$ & $\begin{array}{l}0-6 \text { metros } \\
\text { profundidad }\end{array}$ & $\begin{array}{c}\text { diciembre } 2003 \text {, } \\
\text { junio } 2006 \text {, abril } \\
2007\end{array}$ \\
\hline 3. Sabancuy & $\begin{array}{c}18^{\circ} 59^{\prime} 35^{\prime \prime} \mathrm{N} \\
91^{\circ} 11^{\prime} 08.3^{\prime \prime} \mathrm{W}\end{array}$ & 0-1 metro profundidad & $\begin{array}{c}\text { octubre } 2004, \text { junio } \\
\text { y noviembre } 2006, \\
\text { abril } 2007\end{array}$ \\
\hline $\begin{array}{l}\text { 4. } 29 \mathrm{~km} \text { al NE de } \\
\text { Sabancuy }\end{array}$ & $\begin{array}{c}19^{\circ} 07^{\prime} 50^{\prime \prime} \mathrm{N} \\
90^{\circ} 58^{\prime} 35.6^{\prime \prime} \mathrm{W}\end{array}$ & $\begin{array}{l}\text { 0-3 metros } \\
\text { profundidad }\end{array}$ & $\begin{array}{c}\text { octubre } 2004, \text { junio } \\
\text { y noviembre } 2006, \\
\text { abril } 2007\end{array}$ \\
\hline 5. Punta Xen & $\begin{array}{l}19^{\circ} 10^{\prime} 50^{\prime \prime} \mathrm{N} \\
90^{\circ} 54^{\prime} 9.6^{\prime \prime} \mathrm{W}\end{array}$ & 0-1 metro profundidad & $\begin{array}{c}\text { octubre } 2003, \\
\text { abril } 2004, \text { junio y } \\
\text { noviembre } 2006\end{array}$ \\
\hline 6. Champotón & $\begin{array}{l}19^{\circ} 20^{\prime} 55^{\prime \prime} \mathrm{N} \\
90^{\circ} 43^{\prime} 48^{\prime \prime} \mathrm{W}\end{array}$ & $\begin{array}{l}0-2 \text { metros } \\
\text { profundidad }\end{array}$ & $\begin{array}{c}\text { diciembre } 2003, \\
\text { octubre } 2004, \text { junio } \\
\text { y noviembre } 2006, \\
\text { abril } 2007\end{array}$ \\
\hline 7. Playa Bonita & $\begin{array}{l}19^{\circ} 47^{\prime} 44.3^{\prime \prime} \mathrm{N} \\
90^{\circ} 37^{\prime} 3.8^{\prime \prime} \mathrm{W}\end{array}$ & $\begin{array}{l}0-2 \text { metros } \\
\text { profundidad }\end{array}$ & $\begin{array}{c}\text { octubre } 2004, \text { junio } \\
\text { y noviembre } 2006, \\
\text { abril } 2007\end{array}$ \\
\hline $\begin{array}{l}\text { 8. Escolleras Ciudad de } \\
\text { Campeche }\end{array}$ & $\begin{array}{l}19^{\circ} 51^{\prime} 49.9^{\prime \prime} \mathrm{N} \\
90^{\circ} 31^{\prime} 39.4^{\prime \prime} \mathrm{W}\end{array}$ & $\begin{array}{l}\text { 0-2 metros } \\
\text { profundidad }\end{array}$ & $\begin{array}{c}\text { octubre } 2004, \text { junio } \\
\text { y noviembre } 2006, \\
\text { abril } 2007\end{array}$ \\
\hline
\end{tabular}

canne et al. (2010). La secuencia de la lista florística sigue el orden propuesto por Anagnostidis y Komárek (1988) para Cyanobacteria y el de Wynne (2011) y Guiry y Guiry (2011) para Rhodophyta, Heterokontophyta y Chlorophyta.

La segunda fase consistió en la revisión de los herbarios ENCB y FEZA, donde se obtuvo la información relativa a las especies recolectadas previamente por otros investigadores; también se corrigieron las determinaciones de algunos ejemplares y por último se realizó una revisión bibliográfica de los estudios llevados a cabo en la región litoral de Campeche desde 1958 hasta 2009, con el fin de obtener los registros de algas marinas previamente citados para el litoral del estado y con ello compararlo con los datos obtenidos en el presente estudio. Con la información obtenida se elaboró 


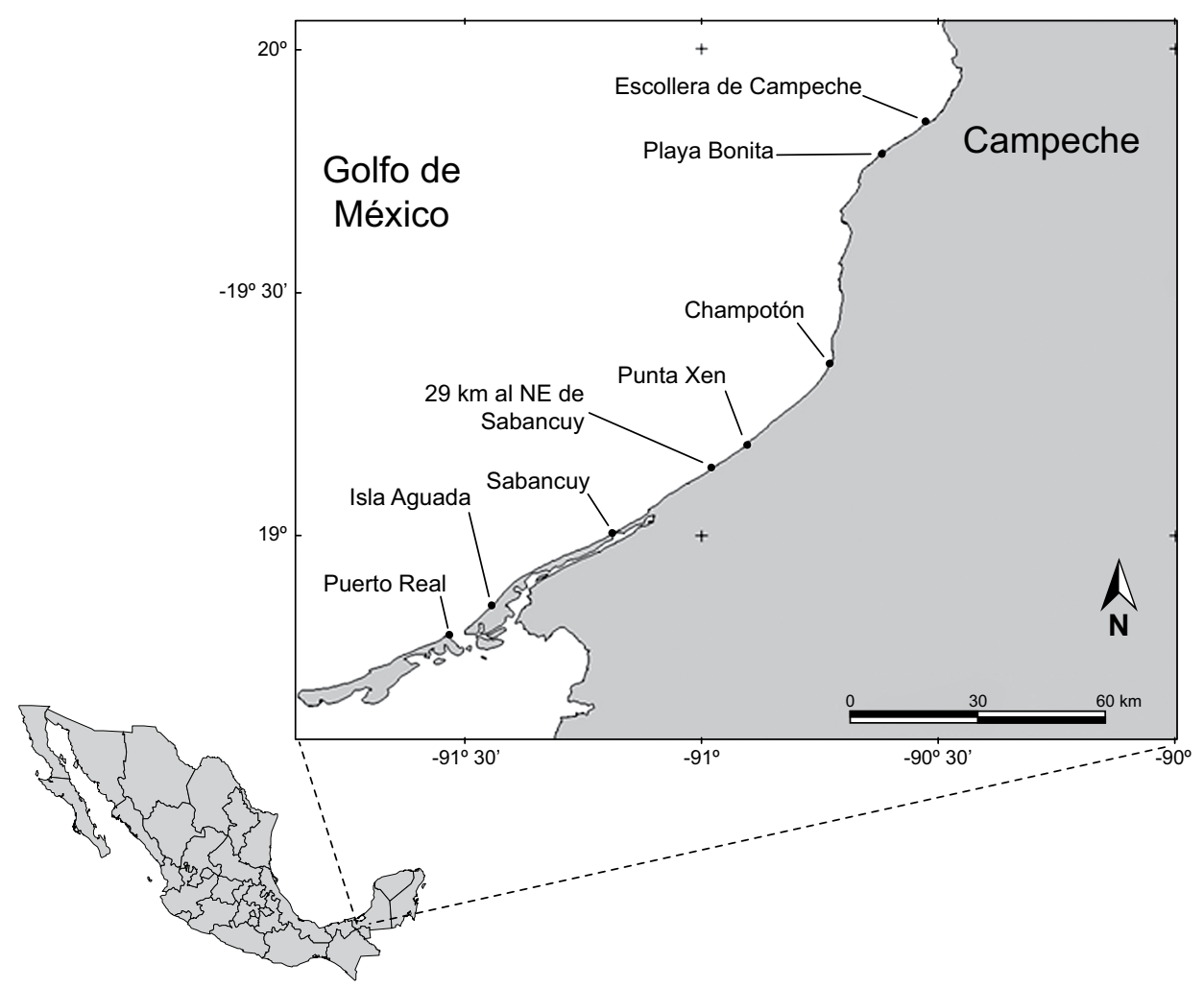

Fig. 1. Ubicación del área de estudio y de las localidades de muestreo.

una lista de especies de algas marinas de la región de Campeche, en la que las familias, géneros y especies están ordenadas alfabéticamente. Para estas últimas se indican datos de distribución en el área de estudio, estado reproductivo, nivel de marea, el modo, hábitat, epifitismo y referencias del origen de registro. La actualización nomenclatural se realizó con el esquema propuesto por Wynne (2011) y Guiry y Guiry (2011).

\section{RESULTADOS Y DISCUSIÓN}

Florística.

De los 211 taxones registrados en este trabajo, destaca la división Rhodophyta con 102 (100 recolectadas por los autores y dos depositadas en el herbario ENCB), 
seguida de Chlorophyta con 66 especies, seis variedades y cuatro formas (68 recolectadas por los autores y nueve depositadas en ENCB), Heterokontophyta con 19 especies (15 recolectadas por los autores y cuatro depositadas en ENCB) y Cyanobacteria con 14. El porcentaje para cada grupo de algas es el siguiente: Cyanobacteria $6.6 \%$, Rhodophyta $48.3 \%$, Heterokontophyta (Phaeophyceae) $9 \%$ y Chlorophyta $36 \%$. En la estación climática de lluvias se presentaron 140 especies y en la de secas 153 (Fig. 2). En la figura 3 se observa el número de taxones por localidad, las barras muestran el número de especies ubicadas por división y por localidad.

Como se observa en el Anexo 2, la mayor riqueza específica se encontró en la época de secas, pues las algas anuales dominan en tal periodo. Este comportamiento de la ficoflora es contrario a lo registrado en los trabajos realizados anteriormente en esta región (Ortega, 1995; Ortega et al., 2001; Callejas-Jiménez et al., 2005), en los que se menciona que es más alta en la época de lluvias.

El grupo con mayor número de especies es el de Rhodophyta, dentro del mismo las familias más diversas son: Rhodomelaceae con 28, Ceramiaceae con 11, Gracilariaceae con 10 y Corallinaceae con nueve. Por lo que respecta a Heterokontophyta (Clase Phaeophyceae), el grupo más numeroso es Dictyotaceae con 14 especies; mientras que en Chlorophyta las más importantes son Caulerpaceae con 18,

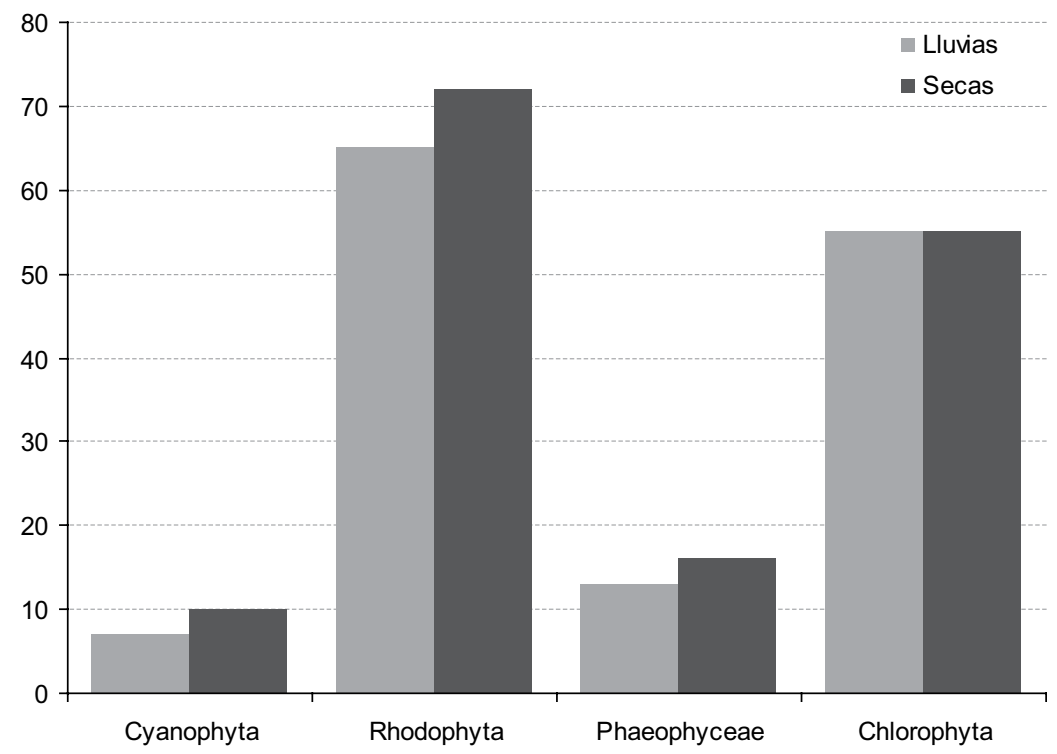

Fig. 2. Total de especies por estación climática. 


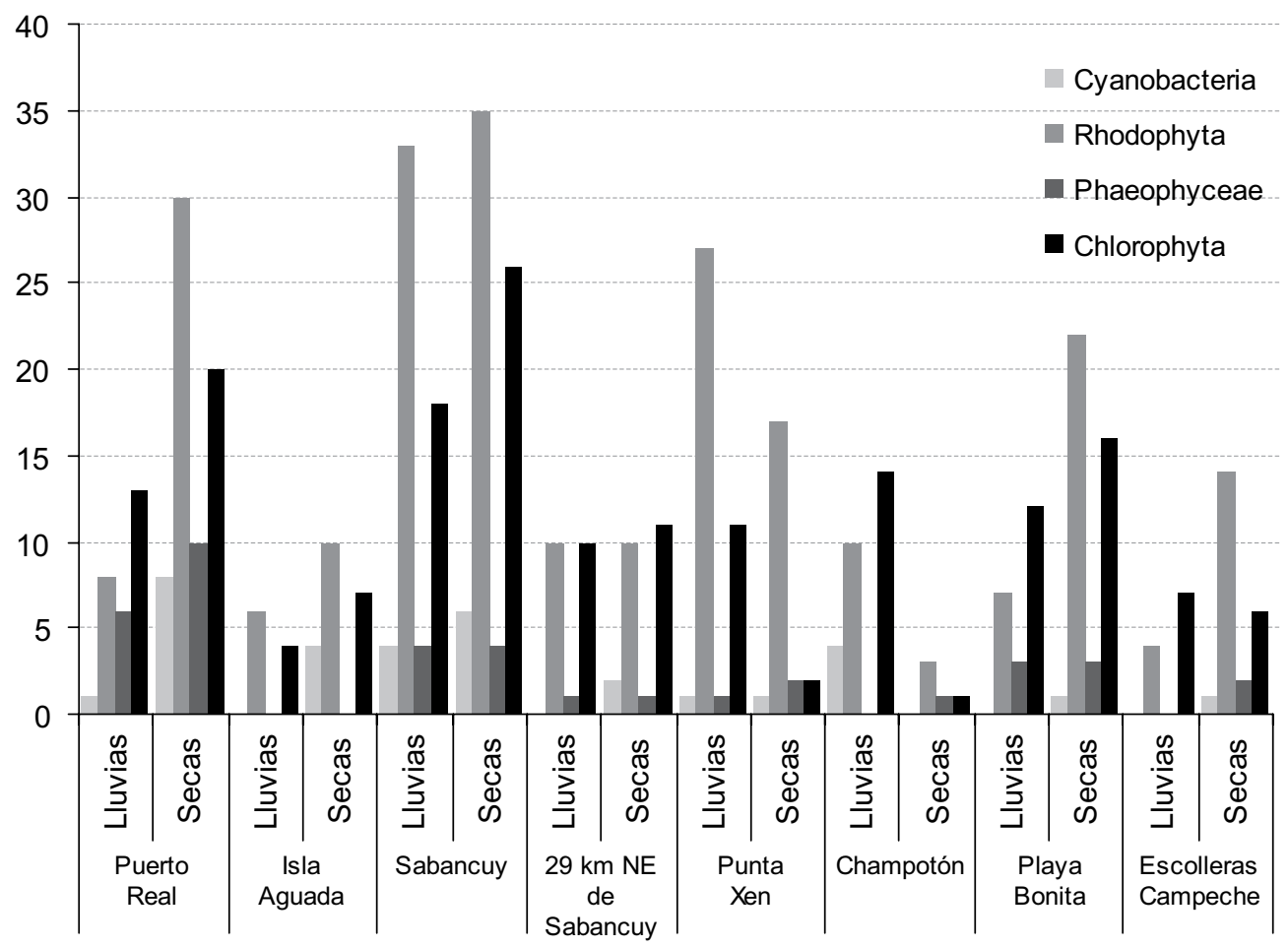

Fig. 3. Total de especies por localidad y por estación climática.

Cladophoraceae con 16 y Udoteaceae con 14. Estas familias en conjunto incluyen $56.9 \%$ del total de la diversidad florística registrada.

La localidad con mayor riqueza corresponde a Sabancuy con 109 especies, seguida por Puerto Real con 86, Playa Bonita con 59 y Punta Xen con 57. Por otro lado, de los 211 taxones enlistados, 30 son nuevos registros para Campeche, mientras que Centroceras micracanthum y Streblonema invisibile lo son para la costa occidental del Atlántico de México (ver Anexo 2). Asimismo, en el Anexo 1 se indican 64 especies conocidas previamente para el litoral de Campeche y que no fueron ubicadas en el presente estudio. Esta circunstancia puede obedecer a varios factores, entre ellos el hecho de que la población humana se ha incrementado notablemente en la zona litoral del estado, además de la construcción de muelles y marinas, que modifican la fisiografía de las playas. Es probable también que algunas especies hayan sido excluidas por el tipo y número de muestreos realizados. En conclusión, y considerando el presente estudio y los trabajos 
hechos previamente en la región, hay un total de 275 taxones conocidos hasta hoy para la zona litoral de Campeche.

Sustrato.

Los miembros de las familias Corallinaceae, Rhodomelaceae, Dictyotaceae y Cladophoraceae se localizaron en facies rocosas expuestas, hábitat frecuente en la zona de estudio. Por lo que respecta a Ceramiaceae sus representantes son epifitas y se ubicaron en numerosos hospederos como algas o pastos marinos. En contraste, las Chlorophyta se desarrollan principalmente como psamófilas; el ambiente arenoso está bien representado en nuestras localidades y la mayoría de las Chlorophyta habitan en este sustrato. Tales resultados concuerdan con los obtenidos por Mateo-Cid y Mendoza-González (2007) y Ortegón-Aznar et al. (2001, 2009).

Biogeografía.

Para el estudio de la ficogeografía descriptiva, Feldmann(1937) propuso el índice R/P (número de especies de Rhodophyta entre número de especies de Phaeophyta) útil para clasificar la flora de una región determinada en función del gradiente latitudinal. Así, un valor de la relación $\mathrm{R} / \mathrm{P}>4$ se suele encontrar en regiones tropicales, mientras que $\mathrm{R} / \mathrm{P}<2$ corresponde con la ficoflora de regiones templado-frías. Cheney (1977) incluyó a las Chlorophyta (C) en un índice análogo al comentado anteriormente $(\mathrm{R}+\mathrm{C}) / \mathrm{P}$, y comprobó que valores de la relación $(\mathrm{R}+\mathrm{C}) / \mathrm{P}>6$ se obtienen en floras tropicales, en tanto que las de mares templados-fríos tienen índices $<3$.

Los resultados del presente estudio fueron analizados con los índices de Feldmann y Cheney y se compararon con lo encontrado por Mateo-Cid y MendozaGonzález (2007) para Isla Cozumel; Mendoza-González et al. (2007) de Isla Mujeres, Dreckmann et al. (1996) para Puerto Morelos y de Huerta-Múzquiz et al. (1987), Ortegón-Aznar et al. (2001, 2009) y Sánchez-Molina et al. (2007) para el litoral de Yucatán. En el Cuadro 2 se muestran los datos obtenidos de los índices de Feldmann y Cheney aplicados a las cuatro regiones mencionadas; la ficoflora de la zona de estudio es similar a la propia del litoral del estado de Yucatán y con un valor cercano al obtenido para la isla de Cozumel e isla Mujeres, localidades netamente caribeñas. Los índices utilizados nos permiten establecer que las algas marinas del litoral de Campeche tienen una predominante distribución tropical y que de acuerdo con ello se registró la presencia de Hypnea musciformis, Halymenia floresii, Jania adhaerens, Gracilaria mammillaris, Gracilariopsis andersonii, Dictyota menstrualis, Sargassum filipendula, Padina gymnospora, Chaetomorpha antennina, Codium isthmocladum y Ulva lactuca, entre otros. 
Cuadro 2. Índices de Feldmann y Cheney para algunos ambientes marinos del litoral del Golfo de México y Caribe mexicano.

\begin{tabular}{lcc}
\hline Localidad/estado & $\begin{array}{c}\text { Índice Feldmann } \\
(\mathrm{R} / \mathrm{P})\end{array}$ & $\begin{array}{c}\text { Índice de Cheney } \\
(\mathrm{R}+\mathrm{C}) / \mathrm{P}\end{array}$ \\
\hline Isla Mujeres, Quintana Roo & 4.50 & 7.15 \\
Isla Cozumel, Quintana Roo & 4.40 & 7.04 \\
Puerto Morelos, Quintana Roo & 3.40 & 5.60 \\
Litoral del estado de Yucatán & 6.00 & 9.00 \\
Litoral de Campeche (este estudio) & 5.37 & 9.30 \\
\hline
\end{tabular}

Reproducción.

La fase reproductiva que se encontró con mayor frecuencia en este estudio fue la tetraspórica, la que superó tanto a las algas encontradas con fase gamética como a las que se observaron con células de resistencia (heterocistos, gonidangios, acinetos). Estos datos nos permiten considerar que esta generación tiene una supervivencia más larga con respecto a la gametofítica, y a otros mecanismos de reproducción como la propagación vegetativa o la apomeiosis (Ardito y Gómez, 2005). De los 211 taxones ubicados en este estudio, $82 \%$ presentaron estructuras reproductoras, siendo las algas Rhodophyta el grupo dominante, pues $89 \%$ de sus representantes se encontró en etapa fértil y sólo $11 \%$ se halló en estado vegetativo. La más común en Rhodophyta fue la tetraspórica. Estos resultados coinciden con los obtenidos por Mateo-Cid y Mendoza-González (1991, 2007), Mendoza-González y Mateo-Cid (1992) y Lucio y Nunes (2002); además, estos últimos autores mencionan que la dominancia de una fase reproductiva puede estar influenciada por cambios temporales de factores ambientales locales. Con respecto a las especies de Cyanobacteria, éstas presentan exclusivamente estructuras asexuales y sólo $35.7 \%$ se encontró en alguna fase de este tipo.

En cuanto a Heterokontophyta, 95\% de sus representantes se encontró en reproducción. En este grupo, en Streblonema invisibile y Hincksia rallsiae, se observaron estructuras pluriloculares tanto en la época de lluvias como en la de secas. En el caso de las especies de los géneros Padina y Dictyota se ubicaron esporangios, mientras que en el de las de Sargassum se observaron oogonios y anteridios. En contraste, en Chlorophyta solo $13.4 \%$ de los taxones presentó algún estado reproductivo, tal circunstancia puede deberse a que estas algas se encontraban en estadios juveniles y a que las fases sexuales de estos organismos tienen un periodo corto de vida (Santelices, 1977; Littler et al., 1983). 
Especies epifitas.

En conjunto se localizaron 53 especies epifitas (ver Anexo 2), de ellas, 11 corresponden a Cyanobacteria, 36 a Rhodophyta, dos a Phaeophyceae y cuatro a Chlorophyta. El mayor número de epifitas se obtuvo en la estación climática de secas con 45, observándose la mayor cantidad de especies de Cyanobacteria, así como de las familias Rhodomelaceae y Ceramiaceae, las cuales cubren a otras algas hasta casi $90 \%$ de su superficie. Esto concuerda con lo observado en algunas algas pardas y fanerógamas marinas, en las que el nivel de epifitismo es mayor cuando es más grande la superficie y biomasa de estos organismos. Tal condición también se atribuye a la disminución de sustancias de defensa contra las epifitas a medida que el hospedero se vuelve senescente (Ortuño-Aguirre y RiosmenaRodriguez, 2007). Por lo que respecta a la época de lluvias, sólo se ubicaron 27 especies, lo que puede atribuirse a que las poblaciones de macroalgas bentónicas se encuentran en crecimiento y produciendo sustancias bioactivas, por lo que ofrecen menos superficie susceptible de ser colonizada (Mateo-Cid y Mendoza-González, 1991; Ortuño-Aguirre y Riosmena-Rodríguez, 2007; Menezes de Széchy y Faria de Sá, 2008).

La mayoría de las epifitas se encontraron creciendo frecuentemente sobre algas perennes como Padina spp., Sargassum filipendula y Digenea simplex; en este contexto se ha sugerido que la longevidad del huésped debe ser lo suficientemente larga para permitir a estos organismos completar su ciclo vital y que ésta podría ser una razón probable de la ausencia de epifitas en las algas anuales y efímeras (Santelices, 1977). Por otra parte, las hojas laminares del pasto marino Thalassia testudinum proporcionan un sustrato firme para el establecimiento de una gran diversidad de epibiontes (Barrios y Díaz, 2005). En este estudio se ubicaron 15 especies creciendo sobre Thalassia, siendo en su mayoría algas filamentosas de los géneros Ceramium y Polysiphonia, además de coralinas incrustantes como Hydrolithon farinosum y Lithophyllum pustulatum.

\section{CONCLUSIONES}

El análisis y la integración de los estudios previos y los resultados obtenidos en el presente estudio, nos permiten visualizar la importancia de realizar inventarios ficoflorísticos en regiones del litoral mexicano poco trabajadas. Además, este tipo de investigaciones nos permite conocer de manera fidedigna la biodiversidad de algas marinas presentes en nuestro país. Es importante resaltar que a partir de los 
resultados obtenidos en el presente estudio, se sugiere realizar monitoreos a mediano y largo plazo de las poblaciones de algas marinas como una estrategia que nos permita determinar las posibles variaciones de la ficoflora, ya sea por condiciones climáticas naturales tales como huracanes o períodos de lluvias intensos, así como por las actividades de turismo, pesquerías y aumento de la población humana en las zonas costeras.

\section{AGRADECIMIENTOS}

Al Instituto Politécnico Nacional (SIP-20121102 y SIP-20130481) y al Programa de Apoyo a Proyectos de Investigación e Innovación Tecnológica (PAPIT) claves IN-211505 e IN-222910, de la Universidad Nacional Autónoma de México, por facilitar el apoyo económico, las instalaciones y equipo necesario para el desarrollo de este estudio. La primera y segunda autora agradecen la beca otorgada por la Comisión de Operación y Fomento de Actividades Académicas (COFAA). A Deisy Jazmín García López, Ulises Sandoval Rauda y Julio A. Acosta Calderón por el apoyo logístico. A los revisores anónimos que con sus comentarios y sugerencias mejoraron notablemente este manuscrito.

\section{LITERATURA CITADA}

Aguilar-Rosas, L. E., J. Espinoza-Ávalos y R. Aguilar-Rosas. 2001. Distribución de las especies de la familia Udoteaceae (Bryopsidales, Chlorophyta) de la península de Yucatán, México An. Esc. Nal. Cienc. Biol. Méx. 47(1): 99-108.

Anagnostidis, K. y J. Komárek. 1988. Modern approach to the classification system of Cyanophytes. 3. Oscillatoriales. Arch. Hydrobiol., Suppl. 80: 327-472.

Ardito, S. y S. Gómez. 2005. Patrón fenológico de una población de Gelidium serrulatum J. Agardh (Rhodophyta, Gelidiales) en la localidad de Taguao, Estado Vargas, Venezuela. Acta Bot. Venez. 28(1): 101-111.

Barrios, J. y O. Díaz. 2005. Algas epifitas de Thalassia testudinum en el Parque Nacional Mochima, Venezuela. Bol. Centro Invest. Biol. 39(1): 1-14.

Callejas-Jiménez, M. E., A. Sentíes y K. M. Dreckmann. 2005. Macroalgas de Puerto Real, Faro Santa Rosalía y Playa Preciosa, Campeche, México, con algunas consideraciones florísticas y ecológicas para el estado. Hidrobiológica 15(1): 89-96.

Carranza-Edwards, A., M. Gutiérrez-Estrada y R. Rodríguez Torres. 1975. Unidades morfotectónicas continentales de las costas mexicanas. An. Centro Cienc. Mar Limnol. 2(1): 81-88. 
Cheney, D. P. 1977. A new improved ratio for comparing seaweed floras. J. Phycol. 13 (suppl.): $1-13$.

Cho, T. O., S. M. Boo, M. H. Hommersand, C. A. Maggs, L. McIvor y S. Fredericq. 2008. Gayliella gen. nov. in the tribe Ceramieae (Ceramiaceae, Rhodophyta) based on molecular and morphological evidence. J. Phycol. 44(3): 721-738.

Dawes, C. J. y A. C. Mathieson. 2008. The seaweeds of Florida. University Press of Florida. Gainesville, USA. 591 pp.

De la Lanza Espino, G. y J. C. Gómez Rojas. 2004. Características físicas y químicas del Golfo de México. In: Caso, M., I. Pisanty y E. Ezcurra (comp.). Diagnóstico ambiental del Golfo de México. Vol. 1. Secretaría del Medio Ambiente y Recursos Naturales, Instituto Nacional de Ecología, AC. - Harte Research Institute for Gulf of Mexico Studies. México, D.F., México. pp. 105-136.

Dreckmann, K. M., I. Stout y A. Sentíes. 1996. Lista actualizada de las algas marinas bentónicas de Puerto Morelos, Quintana Roo, Caribe mexicano. Polibotánica 3: $1-17$.

Dreckmann, K. M. y G. De Lara-Isassi. 2000. Gracilaria caudata J. Agardh (Gracilariaceae, Rhodophyta) en el Atlántico mexicano. Hidrobiológica 10(2): 125-130.

Feldmann, J. 1937. Recherches sur la végétation marine de la Méditerranée. La Côte des Albères. Rev. Algol. 10: 1-339.

García, E. 1983. Modificaciones al sistema de clasificación climática de Köppen (para adaptarlo a las condiciones de la República Mexicana). Instituto de Geografía, Universidad Nacional Autónoma de México. México, D.F., México. 246 pp.

Guiry, M. D. y G. M. Guiry. 2011. Algaebase versión 4.2. World-wide electronic Publication. National University of Ireland. Galway, Ireland. http:/www.algaebase.org.

Gurgel, C. F. D., S. Fredericq y J. N. Norris. 2003. Gracilariopsis silvana sp. nov., G. hommersandii sp.nov., and G. cata-luziana sp. nov., three new species of Gracilariaceae (Gracilariales, Rhodophyta) from the western Atlantic. Hidrobiológica 13: 57-68.

Huerta-Múzquiz, L. y A. M. Garza-Barrientos. 1966. Algas marinas del litoral del estado de Campeche. Ciencia (México) 24(5,6): 193-200.

Huerta-Múzquiz, L., A. C. Mendoza-González y L. E. Mateo-Cid. 1987. Avance de un estudio de las algas marinas de la península de Yucatán. Phytologia 62(1): 23-53.

Littler, D. S. y M. M. Littler. 2000. Caribbean reef plants. An identification guide to the reef plants of the Caribbean, Bahamas, Florida and Gulf of Mexico. Offshore Graphics. Washington, USA. 542 pp.

Littler, M. M., D. S. Littler y P. R. Taylor. 1983. Evolutionary strategies in a tropical barrier reef system: functional-form groups of marine macroalgae. J. Phycol. 19: 229-237.

Lucio, A. M. y J. M. de C. Nunes. 2002. Aportación al conocimiento fenológico de las rodofíceas marinas de la playa del Guarajuba (Camaçari, Bahía) Brasil. Bot. Complutensis 26: 17-34.

Martin-Lescanne, J., F. Rousseau, B. de Reviers, C. Payri, A. Couloux, C. Cruaud y L. Le Gall. 2010. Phylogenetic analyses of the Laurencia complex (Rhodomelaceae, Ceramiales) support recognition of five genera: Chondrophycus, Laurencia, Osmundea, Palisada and Yuzurua stat. nov. Eur. J. Phycol. 45(1): 51-61. 
Mateo-Cid, L. E y A. C. Mendoza-González. 1991. Algas marinas bénticas de la isla Cozumel, Quintana Roo, México. Acta Bot. Mex. 16: 57-87.

Mateo-Cid, L. E y A. C. Mendoza-González. 2007. Flora ficológica: Diversidad, importancia económica y conservación. In: Mejía-Ortiz, L. M. (ed.). Biodiversidad acuática de la isla de Cozumel. Universidad de Quintana Roo - Plaza y Valdés. México D.F., México. $420 \mathrm{pp}$.

Mateo-Cid, L. E., A. C. Mendoza-González y R. B. Searles. 2003. La tribu Callithamniae (Ceramiaceae, Rhodophyta) en la costa del Atlántico de México. Hidrobiológica 13(1): 39-50.

Mendoza-González, A. C. y L. E. Mateo-Cid. 1992. Algas marinas bentónicas de Isla Mujeres, Quintana Roo, México. Acta Bot. Mex. 19: 37-61.

Mendoza-González, A. C., L. E. Mateo-Cid, R. Aguilar-Rosas y L. E. Aguilar-Rosas. 2000. La familia Sphacelariaceae (Sphacelariales, Phaeophyta) en las costas de México. Polibotánica 11: 21-48.

Mendoza-González, A. C., L. E. Mateo-Cid y R. B. Searles. 2007. Yucatán seaweeds from the offshore waters of Isla Mujeres, Quintana Roo, México. Bot. Mar. 50: 280-287.

Menezes de Széchy, M. T. y D. A. Faria de Sá. 2008. Variacão sazonal do epifitismo por macroalgas em una populacão de Sargassum vulgare C. Agardh (Phaeophyceae, Fucales) da Bahia da Ilha Grande, Rio de Janeiro. Oecol. Bras. 12(2): 299-314.

Ortega, M. M. 1995. Observaciones del fitobentos de la laguna de Términos, Campeche, México. An. Inst. Biol. UNAM. Ser. Bot. 66: 1-36.

Ortega, M. M., J. L. Godínez y G. Garduño Solórzano. 2001. Catálogo de algas bénticas de las costas mexicanas del Golfo de México y Mar Caribe. Comisión Nacional para el Estudio de la Biodiversidad y Universidad Nacional Autónoma de México. México, D.F., México. 594 pp.

Ortegón-Aznar, I., J. González-González y A. Sentíes. 2001. Estudio ficoflorístico de la laguna de Río Lagartos, Yucatán, México. Hidrobiológica 11(2): 97-104.

Ortegón-Aznar, I., I. Sánchez-Molina y R. A. Casanova-Cetz. 2009. The distribution of marine algae in a coastal lagoon, northern Yucatan Peninsula, Mexico. Neotrop. Biol. Conserv. 4(2): 99-105.

Ortuño-Aguirre, C. y R. Riosmena-Rodríguez. 2007. Dinámica en el epifitismo de Padina concrescens (Dictyotales, Phaeophyta) en el sureste de la Península de Baja California, México. Cienc. Mar. 33(3): 311-317.

Pica, Y. G., G. Ponce y M. E. Barrón. 1991. Golfo de México y Mar Caribe. In: De la Lanza, G. (ed.). Oceanografía de mares mexicanos. AGT Editor. México, D.F., México. 580 pp.

Sánchez-Molina, I., J. González-Ceballos, C. Zetina-Morguel y R. Casanova-Cetz. 2007. Análisis de la biodiversidad de algas marinas situadas entre Uaymitún y Chuburná, Yucatán. Ingeniería 11(1): 43-51.

Santelices, B. 1977. Ecología de las algas marinas bentónicas. Universidad Católica de Chile. Santiago de Chile, Chile. 384 pp.

Schneider, C. W. y R. B. Searles. 1991. Seaweeds of the southeastern United States. Cape Hatteras to Cape Cañaveral. Duke University Press. Durham, USA. 563 pp. 
Taylor, W. R. 1960. Marine algae of the eastern tropical and subtropical coasts of the America. University of Michigan Press. Ann Arbor, USA. 870 pp.

Wynne, M. J. 2011. A checklist of benthic marine algae of the tropical and subtropical western Atlantic: third revision. Nova Hedwigia Beih. 140(1-6): 7-66.

Recibido en marzo de 2011.

Aceptado en febrero de 2013. 


\section{ANEXO 1. LISTA DE ESPECIES NO UBICADAS EN ESTE ESTUDIO}

Referencias bibliográficas: C1 - Huerta y Garza-Barrientos (1966); C2 - Huerta-Múzquiz et al. (1987); C3 - Ortega (1995); C4 - Callejas-Jiménez et al. (2005)

\begin{tabular}{|c|c|}
\hline División/Especie & Referencia \\
\hline \multicolumn{2}{|l|}{ Cyanobacteria } \\
\hline \multicolumn{2}{|l|}{ Clase Cyanophyceae } \\
\hline \multicolumn{2}{|l|}{ Orden Oscillatoriales } \\
\hline \multicolumn{2}{|l|}{ Oscillatoriaceae } \\
\hline 1. Lyngbya aestuarii (Mertens) Liebmann ex Gomont & $\mathrm{C} 3$ \\
\hline \multicolumn{2}{|l|}{ Rivulariaceae } \\
\hline 2. Calothrix longifila W.R. Taylor & $\mathrm{C} 3$ \\
\hline \multicolumn{2}{|l|}{ Clase Compsopogonophyceae } \\
\hline \multicolumn{2}{|l|}{ Orden Erythropeltidales } \\
\hline \multicolumn{2}{|l|}{ Erythrotrichiaceae } \\
\hline 3. Erythrocladia irregularis Rosenvinge & $\mathrm{C} 3$ \\
\hline \multicolumn{2}{|l|}{ Clase Florideophyceae } \\
\hline \multicolumn{2}{|l|}{ Orden Acrochaetiales } \\
\hline \multicolumn{2}{|l|}{ Acrochaetiaceae } \\
\hline 4. Acrochaetium hypneae (Børgesen) Børgesen & $\mathrm{C} 3$ \\
\hline \multicolumn{2}{|l|}{ Orden Corallinales } \\
\hline \multicolumn{2}{|l|}{ Corallinaceae } \\
\hline 5. Amphiroa vanbossea Lemoine & $\mathrm{C} 2$ \\
\hline 6. Hydrolithon boreale (Foslie) Y.M. Chamberlain & $\mathrm{C} 3$ \\
\hline 7. Jania pumila J.V. Lamouroux & $\mathrm{C} 4$ \\
\hline \multicolumn{2}{|l|}{ Orden Ceramiales } \\
\hline \multicolumn{2}{|l|}{ Callithamniaceae } \\
\hline 8. Aglaothamnion cordatum (Borgesen) Feldmann-Mazoyer & $\mathrm{C} 1, \mathrm{C} 2$ \\
\hline \multicolumn{2}{|l|}{ Ceramiaceae } \\
\hline 9. Centroceras clavulatum (C. Agardh) Montagne & $\mathrm{C} 1, \mathrm{C} 2, \mathrm{C} 3, \mathrm{C} 4$ \\
\hline 10. Ceramium diaphanum (Lightfoot) Roth & $\mathrm{C} 3$ \\
\hline 11. C. tenuissimum (Lyngbye) J. Agardh & $\mathrm{C} 1, \mathrm{C} 2$ \\
\hline \multicolumn{2}{|l|}{ Delesseriaceae } \\
\hline 12. Taenioma perpusillum (J. Agardh) J. Agardh & $\mathrm{C} 4$ \\
\hline \multicolumn{2}{|l|}{ Rhodomelaceae } \\
\hline 13. Bryocladia cuspidata (J. Agardh) De Toni & $\mathrm{C} 2, \mathrm{C} 4$ \\
\hline
\end{tabular}


Anexo 1. Continuación.

\begin{tabular}{ll}
\hline División/Especie & Referencia \\
\hline 14. Chondria capillaris Hudson & $\mathrm{C} 4$ \\
15. C. sedifolia Harvey & $\mathrm{C} 3$ \\
16. C. tenuissima (Withering) C. Agardh & $\mathrm{C} 3$ \\
17. Lophocladia trichoclados (C. Agardh) J. Agardh & $\mathrm{C} 3$ \\
18. Polysiphonia foetidissima Cocks ex Bornet & $\mathrm{C} 2$ \\
19. P. hapalacantha Harvey & $\mathrm{C} 2$ \\
20. Yuzurua poiteaui var. gemmifera (Harvey) M.J. Wynne & $\mathrm{C} 3$
\end{tabular}

\section{Orden Gelidiales}

\section{Gelidiaceae}

21. Gelidium americanum (W.R. Taylor) Santelices C2, C3

\section{Orden Gigartinales}

Caulacanthaceae

22. Catenella caespitosa (Whitering) L.M. Irvine

Cystocloniaceae

23. Hypnea cornuta (Kützing) J. Agardh $\quad \mathrm{C} 2$

24. H. valentiae (Turner) Montagne C3

Kallymeniaceae

25. Kallymenia limminghei Montagne

Phyllophoraceae

26. Gymnogongrus crenulatus (Turner) J. Agardh $\quad \mathrm{C} 1$

27. G. griffithsiae (Turner) Martius C1, C2

28. G. tenuis J. Agardh $\quad \mathrm{C} 1, \mathrm{C} 2, \mathrm{C} 3$

Solieriaceae

29. Agardhiella ramosissima (Harvey) Kylin C1, C2, C3

30. Kappaphycus inermis (F. Schmitz) Doty ex H.D. Nguyen et Q.N. C3 Huynh

\section{Orden Gracilariales}

\section{Gracilariaceae}

31. Gracilaria armata (C. Agardh) Greville

$\mathrm{C} 2$

32. G. domingensis (Kützing) Sonder ex Dickie C3

33. G. foliifera (Forsskål) Børgesen $\quad \mathrm{C} 1, \mathrm{C} 2$

34. G. gracilis (Stackhouse) M. Steentoft, L.M. Irvine et W.F. Farnham C1, C3

35. G. venezuelensis W.R. Taylor C3

36. Gracilariopsis lemaneiformis (Bory de Saint-Vincent) E.Y. Dawson, C3 Acleto et Foldvik 
Anexo 1. Continuación.

\begin{tabular}{lc}
\hline División/Especie & Ref \\
\hline 37. Hydropuntia caudata (J. Agardh) Gurgel et Fredericq & C3 \\
Orden Halymeniales & \\
Halymeniaceae & $\mathrm{C} 1$ \\
38. Grateloupia filicina (J.V. Lamouroux) C. Agardh &
\end{tabular}

Orden Rhodymeniales

Champiaceae

39. Lomentaria baileyana (Harvey) Farlow

Orden Nemastomatales

Nemastomataceae

40. Nemastoma gelatinosum M.A. Howe

C1

\section{HETEROKONTOPHYTA}

Clase Phaeophyceae

Orden Dictyotales

Dictyotaceae

41. Dictyota crenulata J. Agardh $\quad$ C4

Orden Ectocarpales

Acinetosporaceae

42. Feldmannia indica (Sonder) Womersley et A. Bailey C3

43. Hincksia mitchelliae (Harvey) P.C. Silva C1, C3

\section{Ectocarpaceae}

44. Asterocladon rhodochortonoides (Børgesen) Uwai, Nagasato, Motomura et Kogame

Orden Scytosiphonales

Scytosiphonaceae

45. Colpomenia sinuosa (Mertens ex Roth) Derbès et Solier

Orden Fucales

Sargassaceae

46. Sargassum vulgare C. Agardh

C4

47. Turbinaria turbinata (Linnaeus) Kuntze

$\mathrm{C} 1, \mathrm{C} 2$

\section{CHLOROPHYTA}

Clase Ulvophyceae

Orden Ulvales

Kornmanniaceae

48. Blidingia minima (Nägeli ex Kützing) Kylin

C1 
Anexo 1. Continuación.

\begin{tabular}{ll}
\hline División/Especie & Referenci \\
\hline Ulvaceae & \\
49. Ulva flexuosa subsp. paradoxa (C. Agardh) M.J. Wynne & C2, C3 \\
50. U. prolifera O.F. Müller & C1
\end{tabular}

\section{Ulvellaceae}

51. Acrochaete viridis (Reinke) R. Nielsen

C3

52. Entocladia ventriculosa (Børgesen) W.R. Taylor

C3

Orden Cladophorales

\section{Cladophoraceae}

53. Chaetomorpha gracilis Kützing

C1, C2

54. C. minima Collins et Hervey

C1

55. Cladophora prolifera (Roth) Kützing

C4

\section{Orden Bryopsidales}

\section{Bryopsidaceae}

56. Bryopsis ramulosa Montagne

\section{Codiaceae}

57. C. isthmocladum subsp. clavatum (Collins et Hervey) P.C. Silva

C1

\section{Caulerpaceae}

58. Caulerpa cupressoides var. serrata (Kützing) Weber-van Bosse

$\mathrm{C} 2$

59. C. cupressoides var. turneri Weber-van Bosse

$\mathrm{C} 1, \mathrm{C} 2$

60. C. mexicana f. pectinata (Kützing) W.R. Taylor

$\mathrm{C} 3$

61. C. prolifera f. obovata J. Agardh

$\mathrm{C} 1, \mathrm{C} 2$

62. C. prolifera f. zosterifolia Børgesen

C3

63. Caulerpa sertularioides f. corymbosa W.R. Taylor

C3

Udoteaceae

64. Udotea unistratea D.S. Littler et M.M. Littler

C4 


\title{
ANEXO 2. ALGAS MARINAS BENTÓNICAS DEL LITORAL DE CAMPECHE
}

\author{
Simbología
}

Localidades:

1 - Puerto Real

5 - Punta Xen

2 - Isla Aguada

6 - Champotón

3 - Sabancuy

4- $29 \mathrm{~km}$ al NE de Sabancuy

7 - Playa Bonita

8 - Escolleras Ciudad de Campeche

Presencia:

L1 - Lluvias (octubre 2004, junio 2006)

S - Secas (diciembre 2003, noviembre 2006, abril 2007)

Reproducción asexual:

Esp - Esporangios

Go - Gonidangios

$\mathrm{Ht}$ - Heterocistos

Hm - Hormogonios

Mn - Monosporas

Plu - Plurangios

Sv - Segregación vegetativa

$\mathrm{Te}$ - Tetrasporangios

Ve - Vegetativo

Habitat:

$$
\begin{aligned}
& \text { Arl - Areno-limoso } \\
& \text { Ar - Arenoso } \\
& \text { End - Endófita } \\
& \text { Epi - Epifito }
\end{aligned}
$$

$$
\begin{aligned}
& \text { Reproducción sexual: } \\
& \text { o - Cistocarpos, oogonios } \\
& \text { o - Gametangios } \\
& \text { Gm - Gametos masculinos } \\
& \text { Zg - Zigosporangios }
\end{aligned}
$$

Modo:

Ex - Expuesto

P - Protegido

Epifita de:

Los números que se indican en esta columna corresponden a las algas o pasto marino (pm) sobre los que se localizaron las especies epifitas.

Observaciones:

Indica la fuente de origen de cada especie:

A - Recolectada por los autores

B - Depositada en herbario ENCB

NRC - Nuevo registro para Campeche

NRM - Nuevo registro México 
Mateo-Cid et al.: Algas marinas bentónicas del litoral de Campeche, México

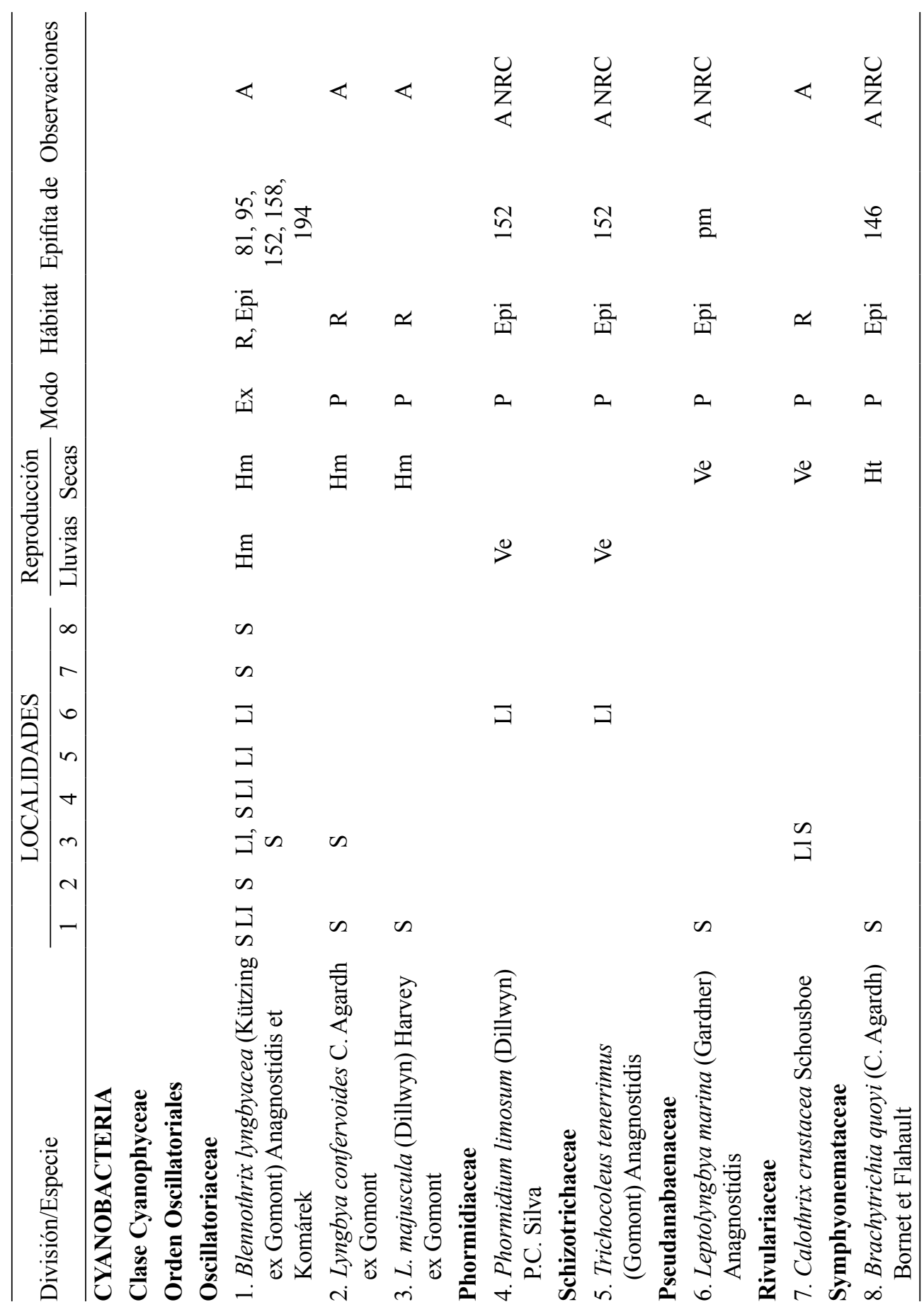


Acta Botanica Mexicana 104: 53-92 (2013)




Mateo-Cid et al.: Algas marinas bentónicas del litoral de Campeche, México

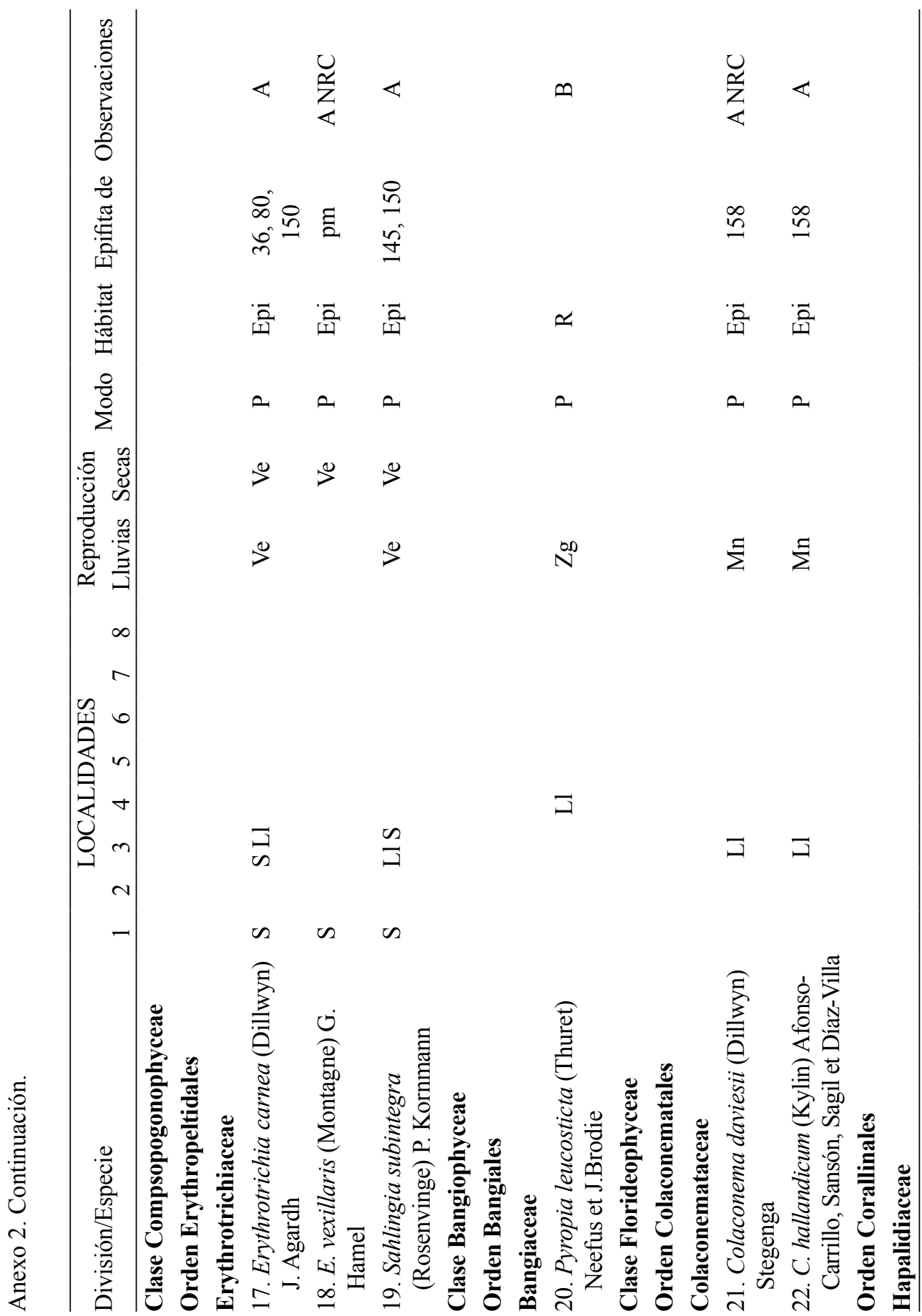


Acta Botanica Mexicana 104: 53-92 (2013)

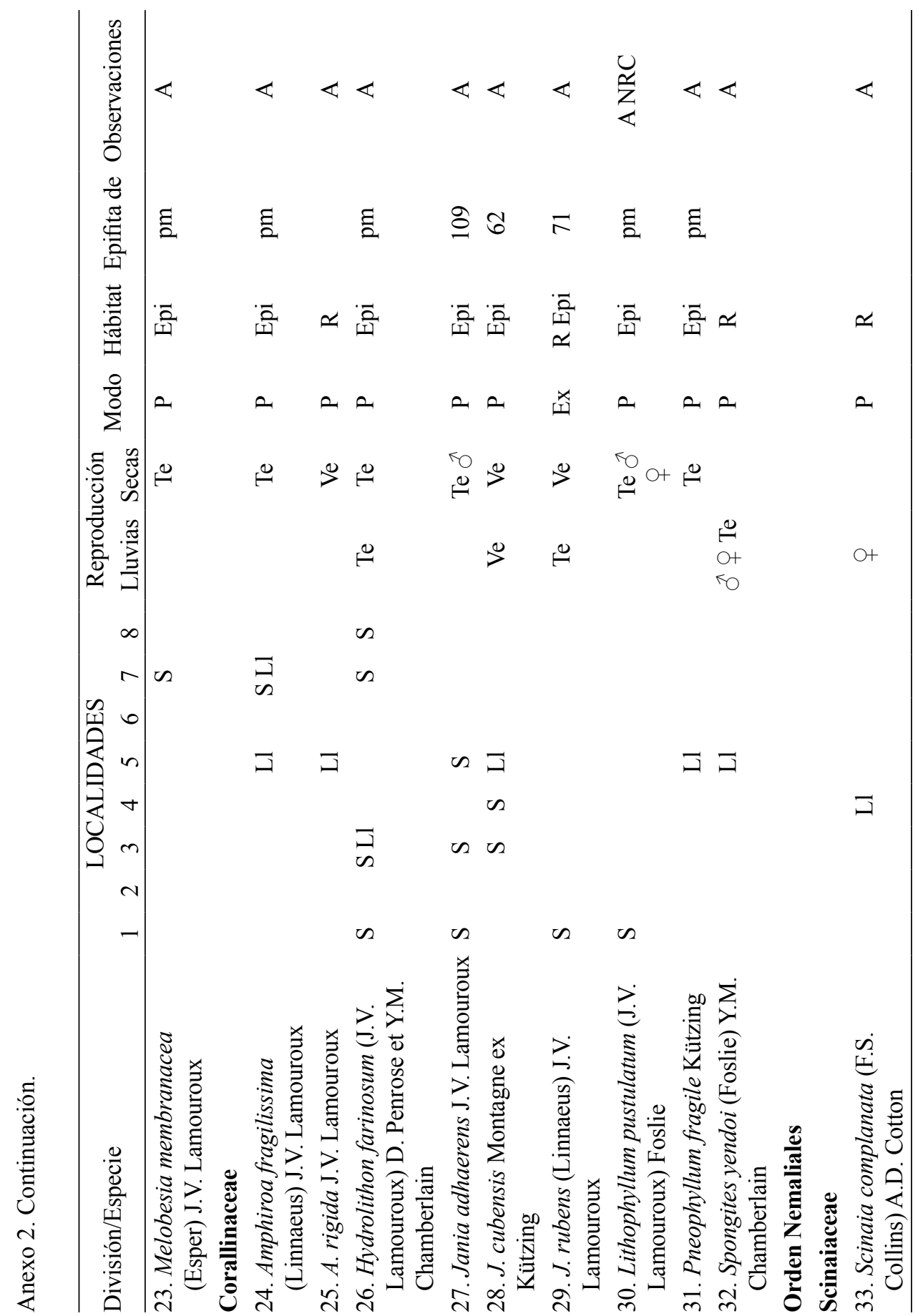


Mateo-Cid et al.: Algas marinas bentónicas del litoral de Campeche, México

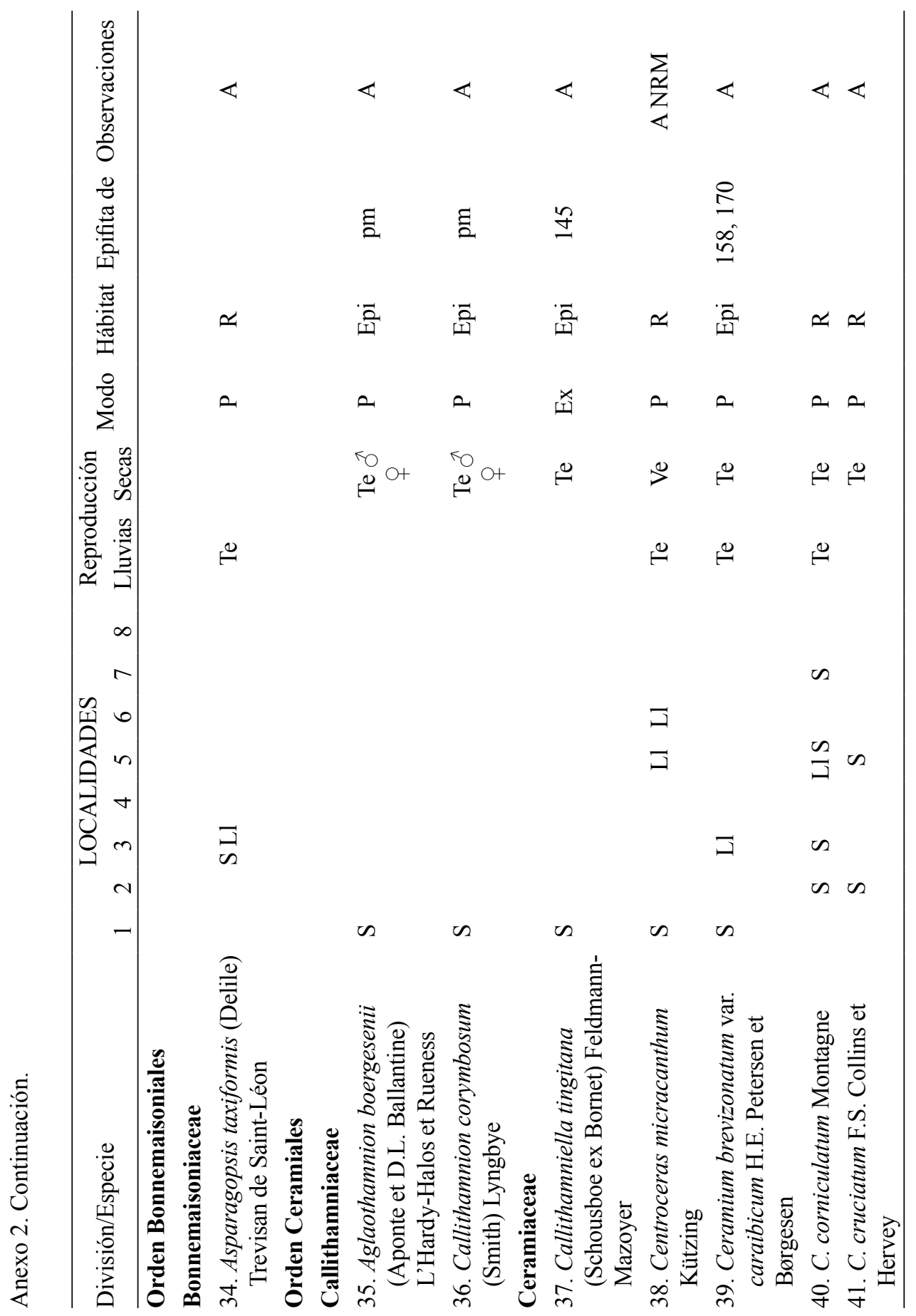


Acta Botanica Mexicana 104: 53-92 (2013)




Mateo-Cid et al.: Algas marinas bentónicas del litoral de Campeche, México

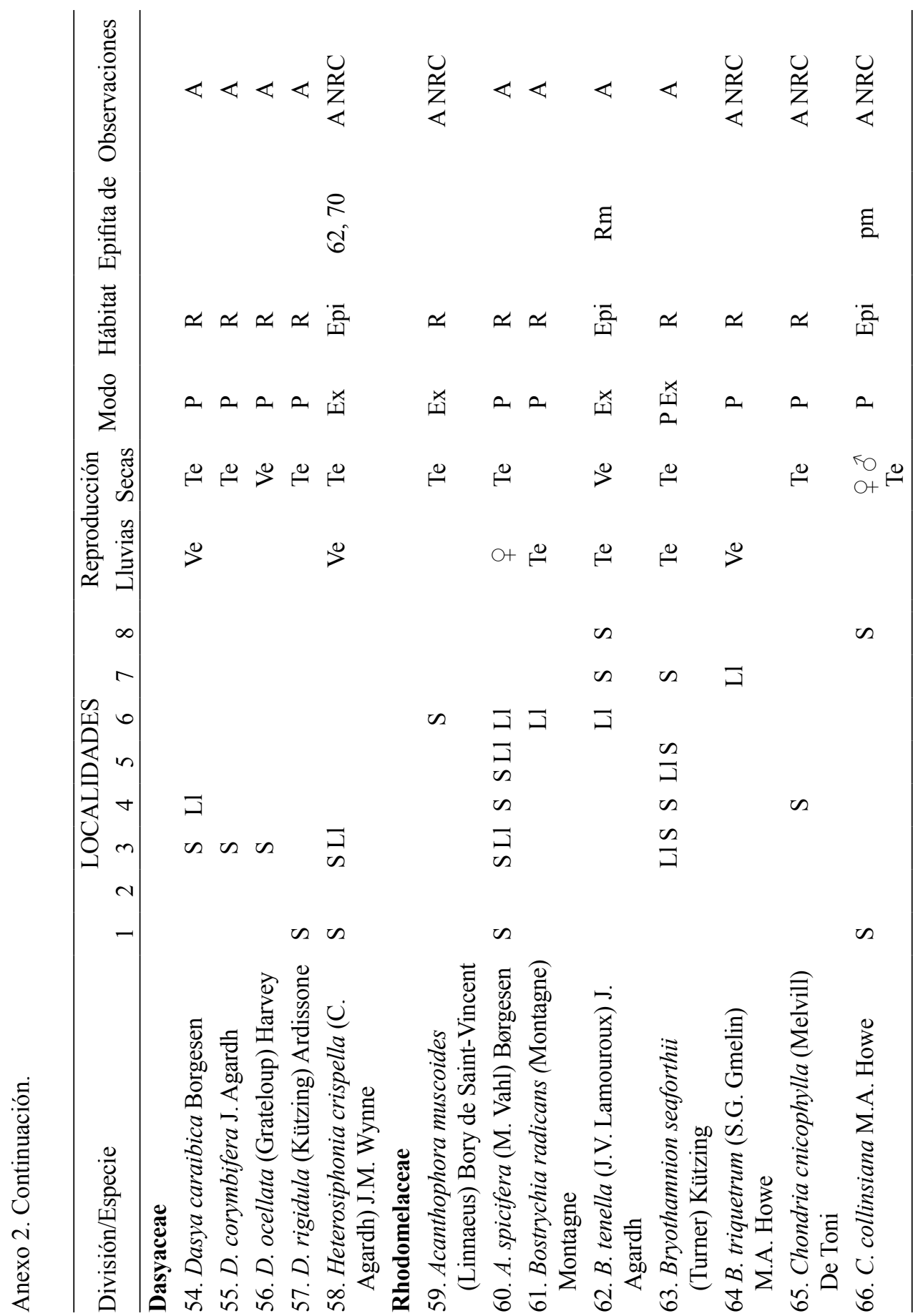


Acta Botanica Mexicana 104: 53-92 (2013)

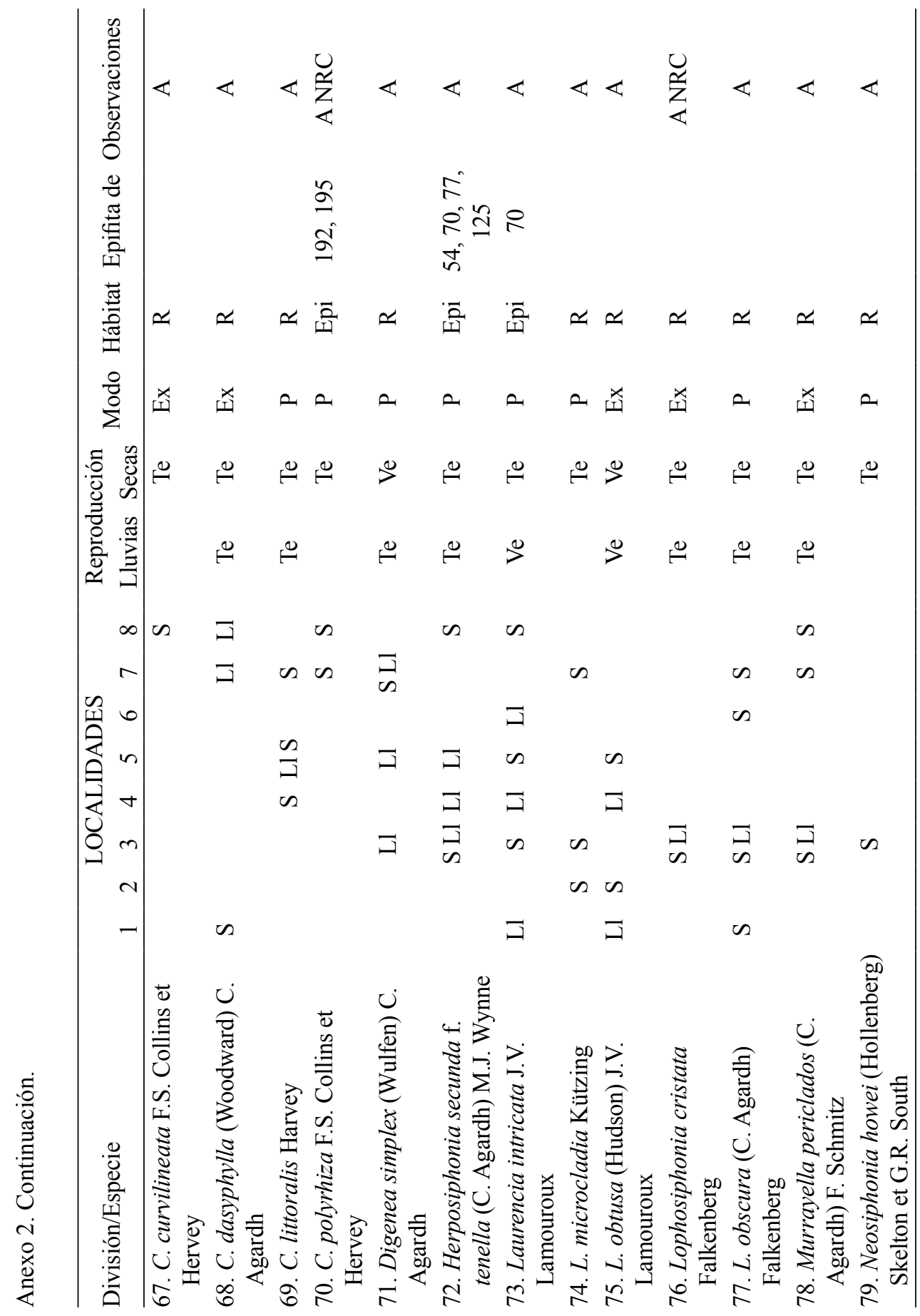


Mateo-Cid et al.: Algas marinas bentónicas del litoral de Campeche, México

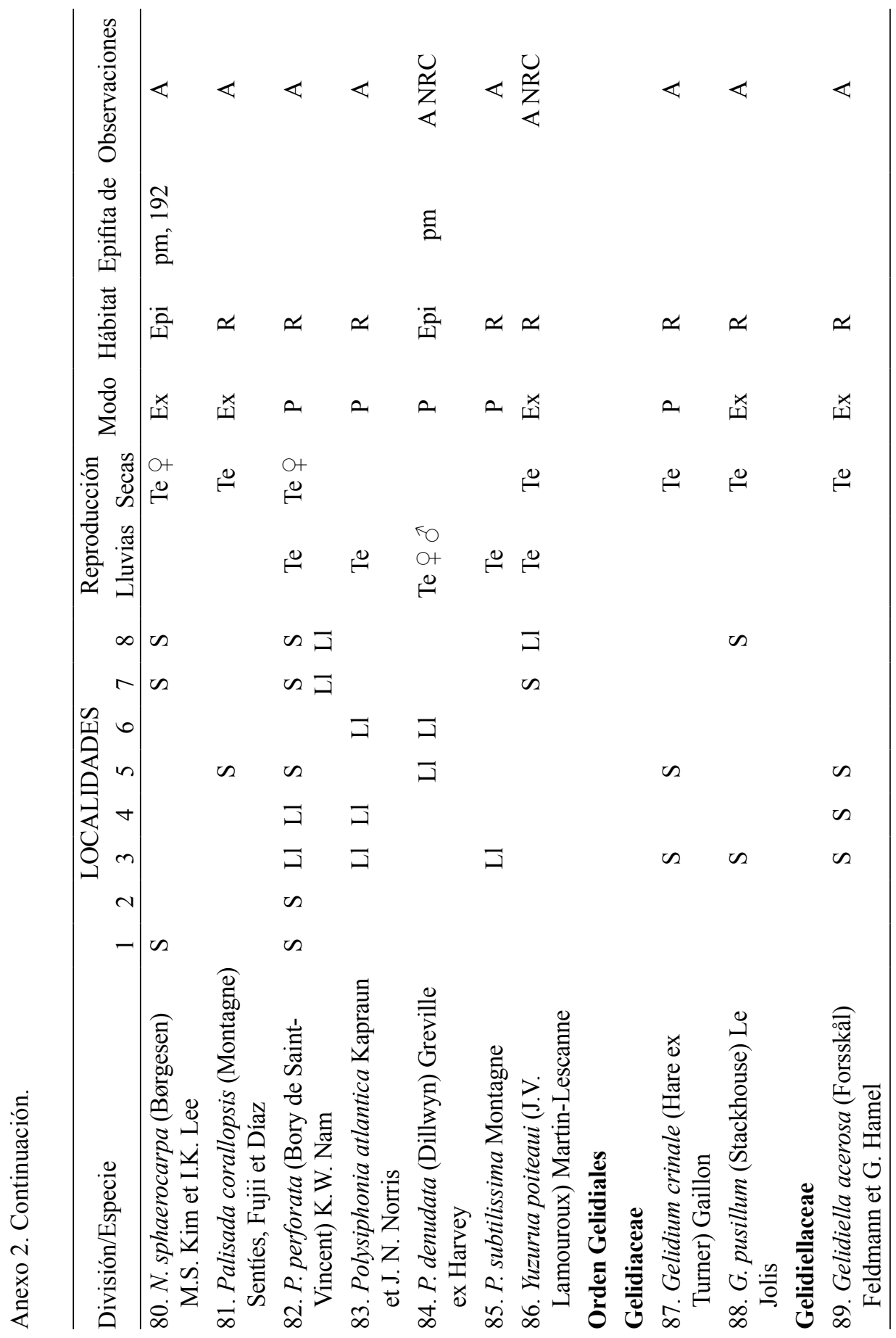


Acta Botanica Mexicana 104: 53-92 (2013)

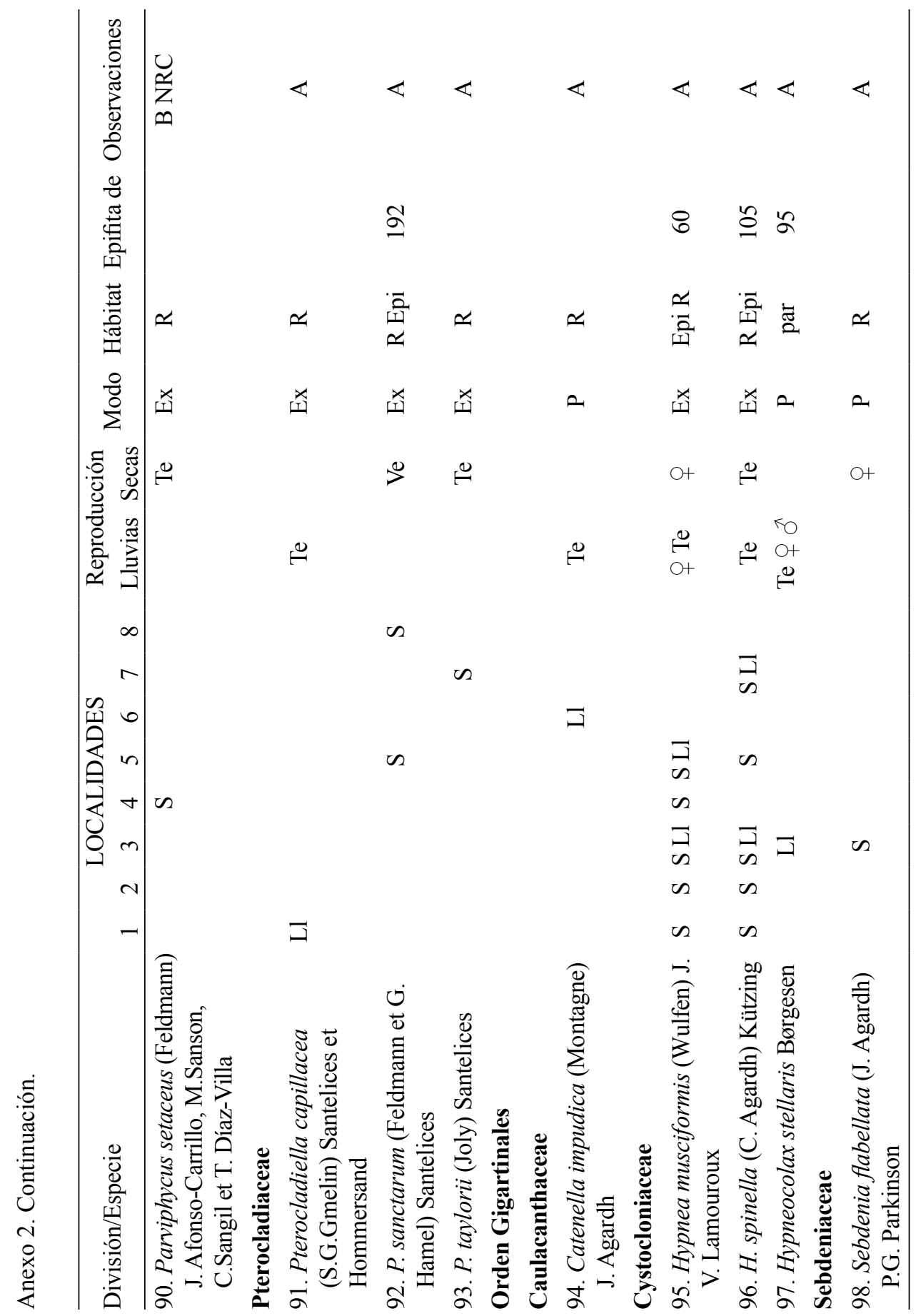


Mateo-Cid et al.: Algas marinas bentónicas del litoral de Campeche, México

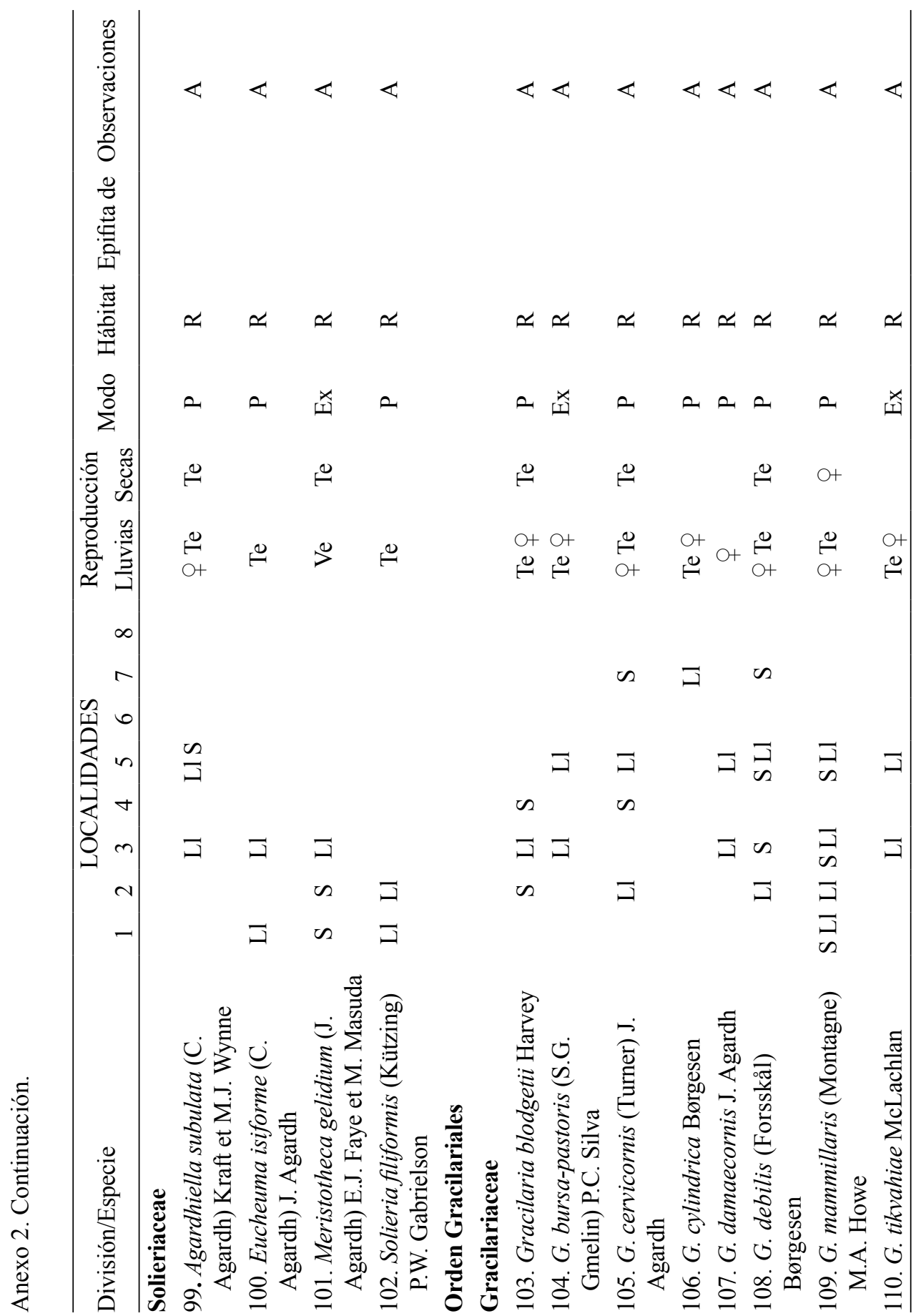


Acta Botanica Mexicana 104: 53-92 (2013)

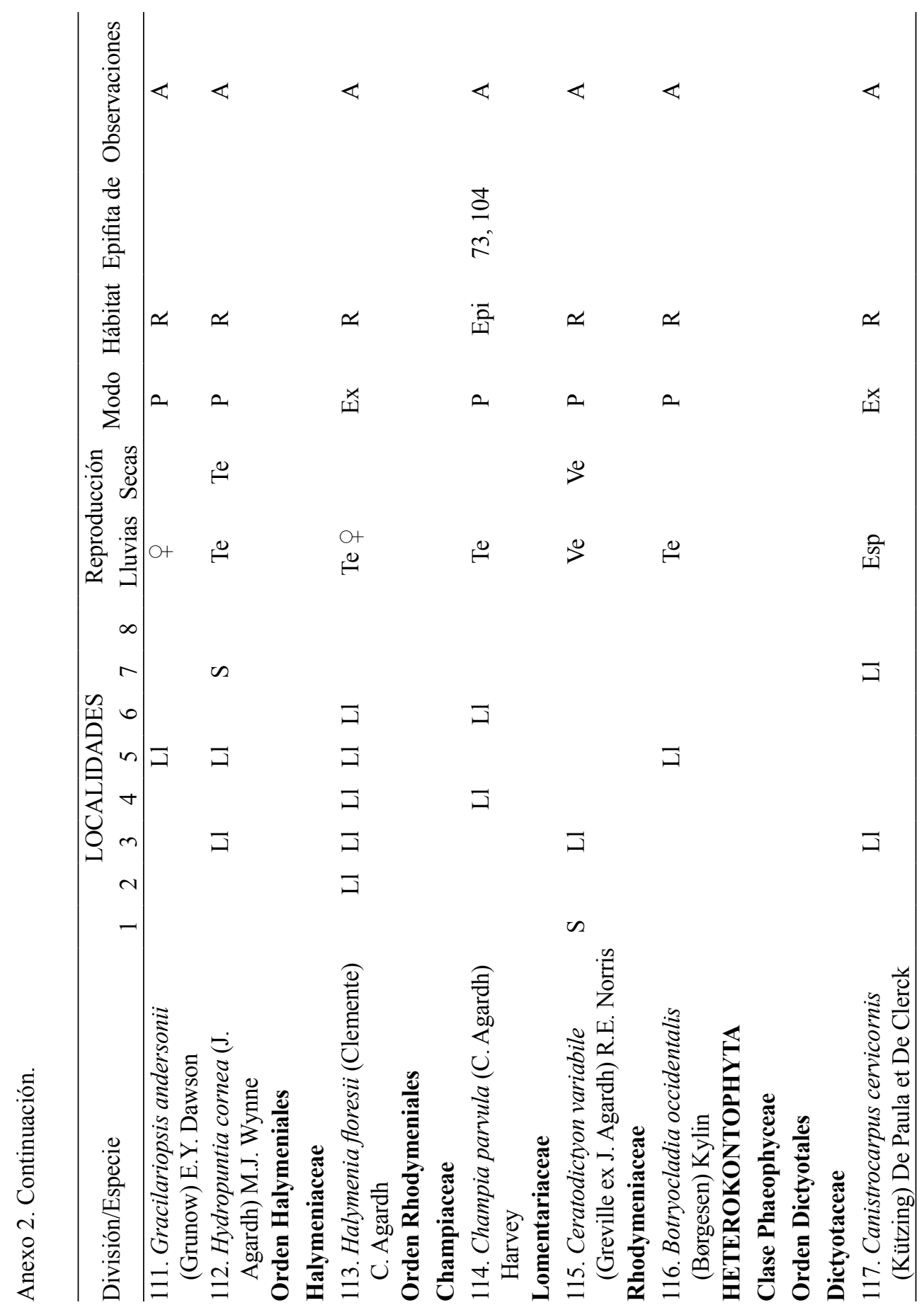


Mateo-Cid et al.: Algas marinas bentónicas del litoral de Campeche, México

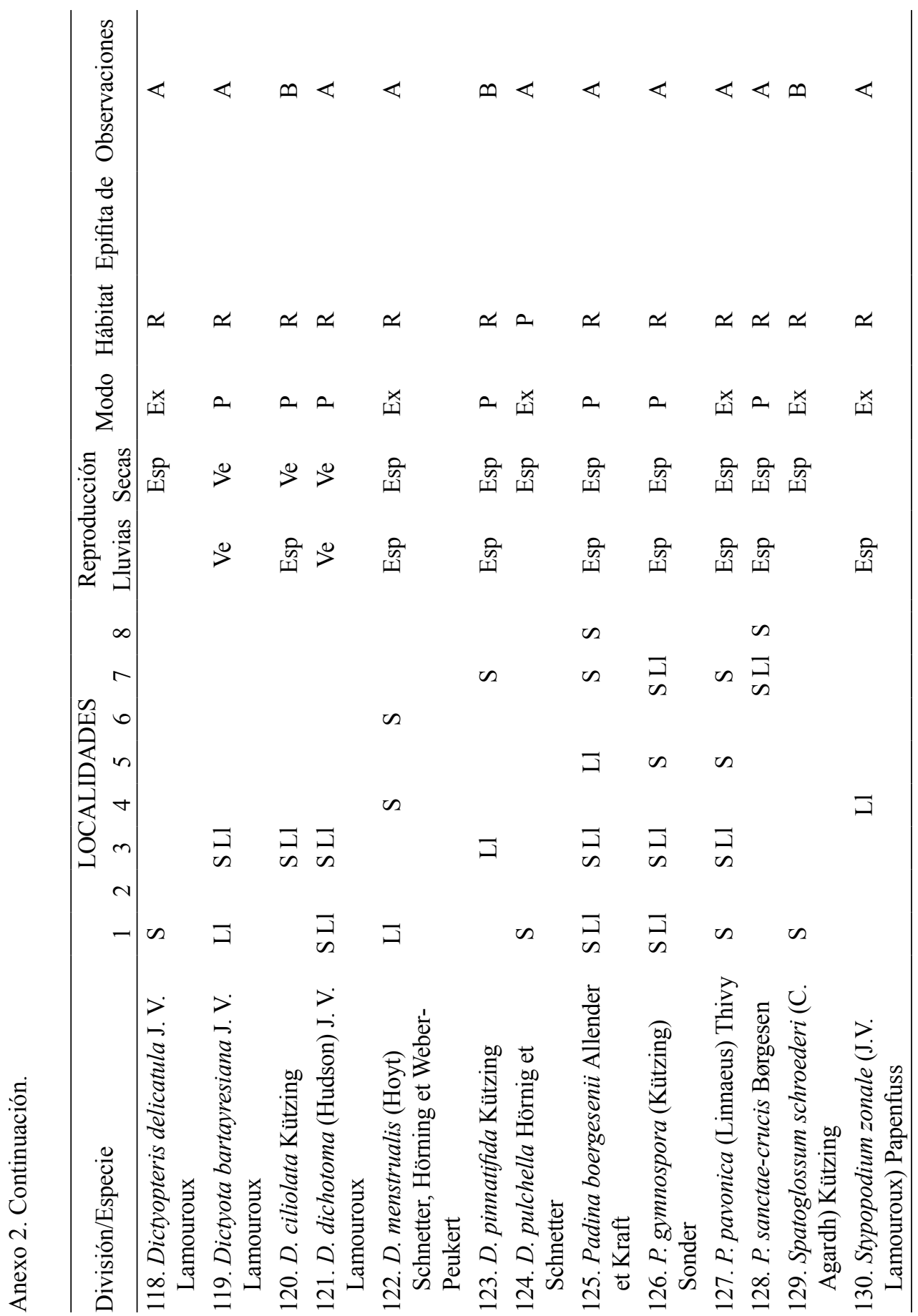


Acta Botanica Mexicana 104: 53-92 (2013)

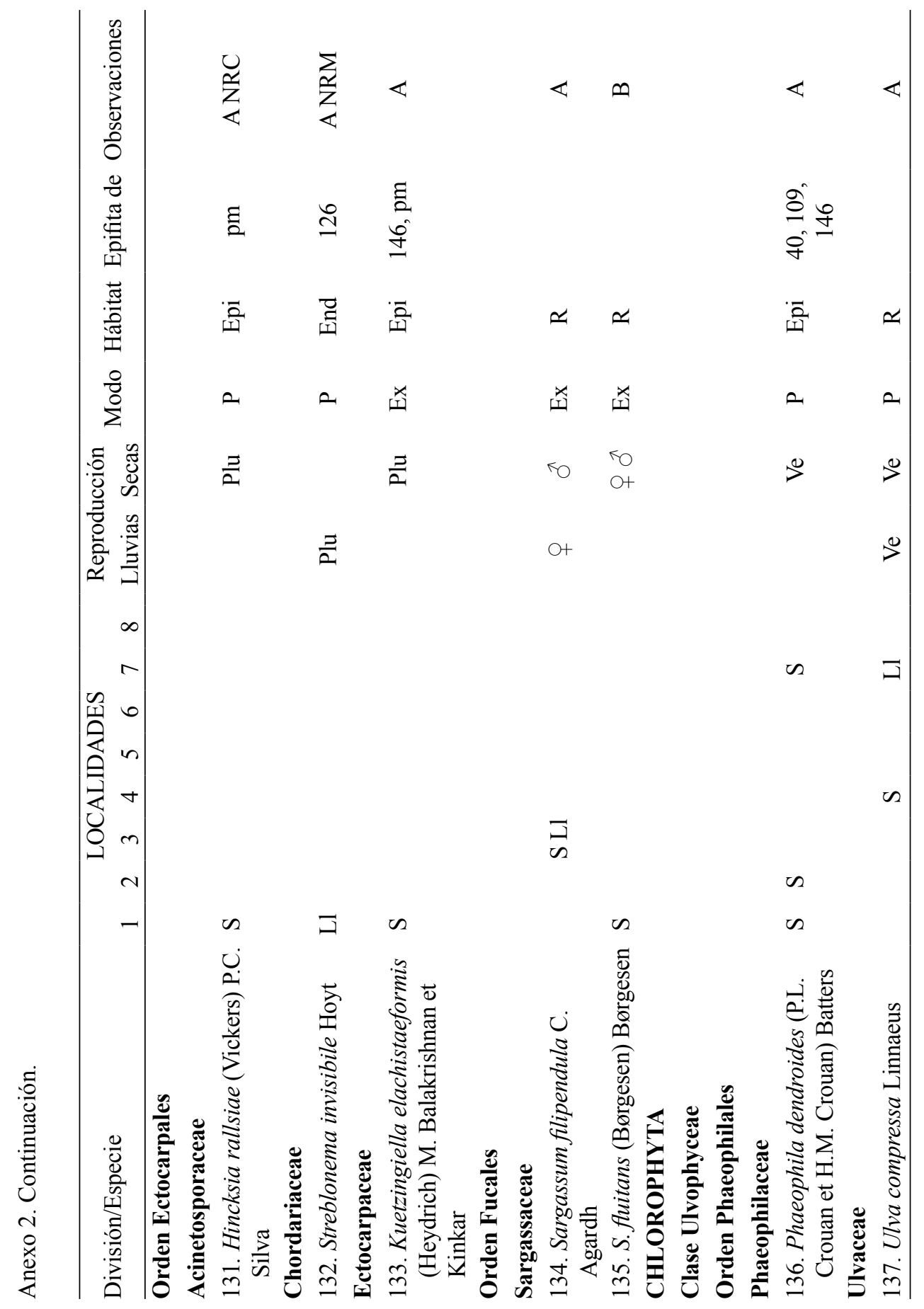


Mateo-Cid et al.: Algas marinas bentónicas del litoral de Campeche, México

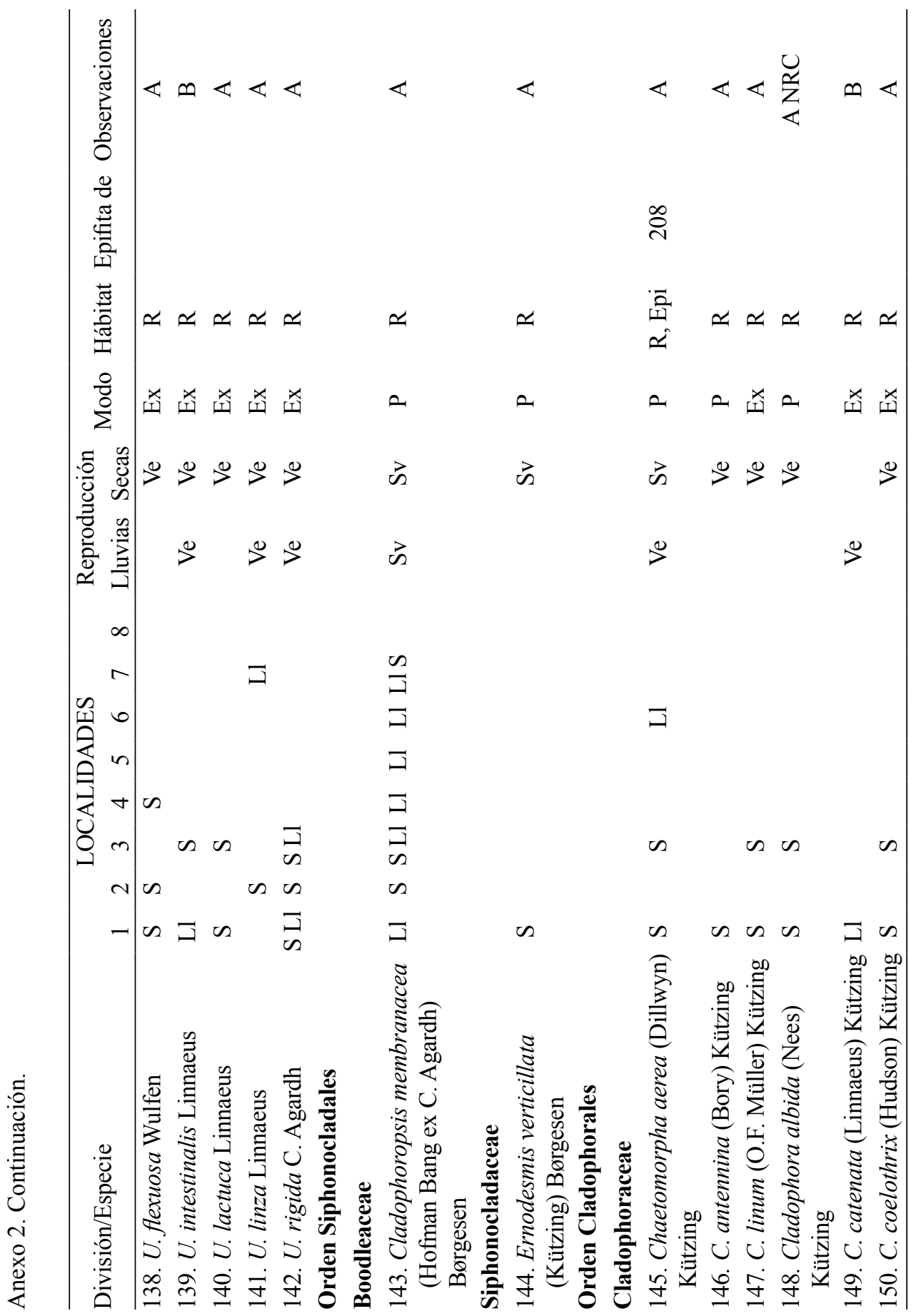


Acta Botanica Mexicana 104: 53-92 (2013)

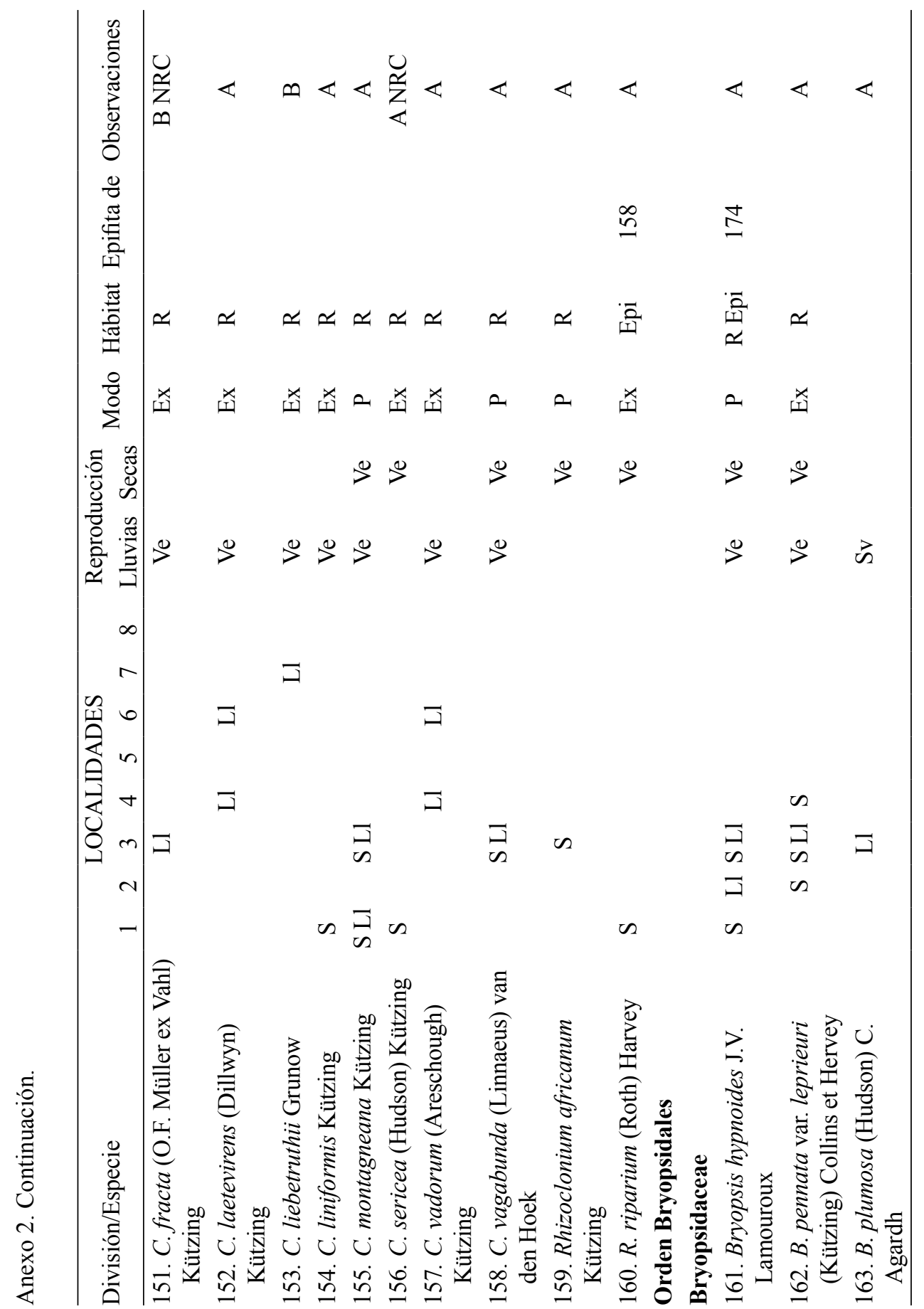


Mateo-Cid et al.: Algas marinas bentónicas del litoral de Campeche, México

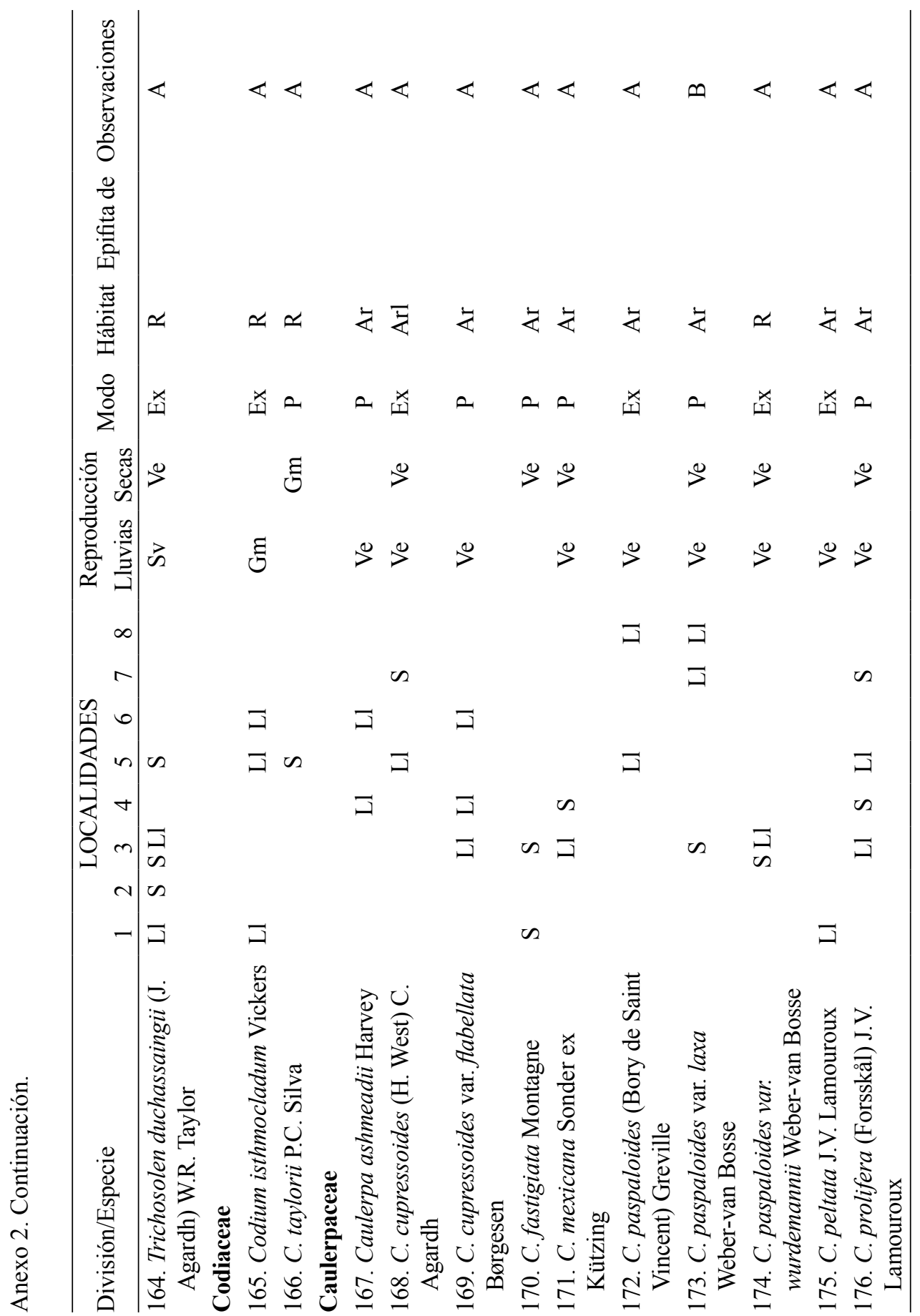


Acta Botanica Mexicana 104: 53-92 (2013)




Mateo-Cid et al.: Algas marinas bentónicas del litoral de Campeche, México

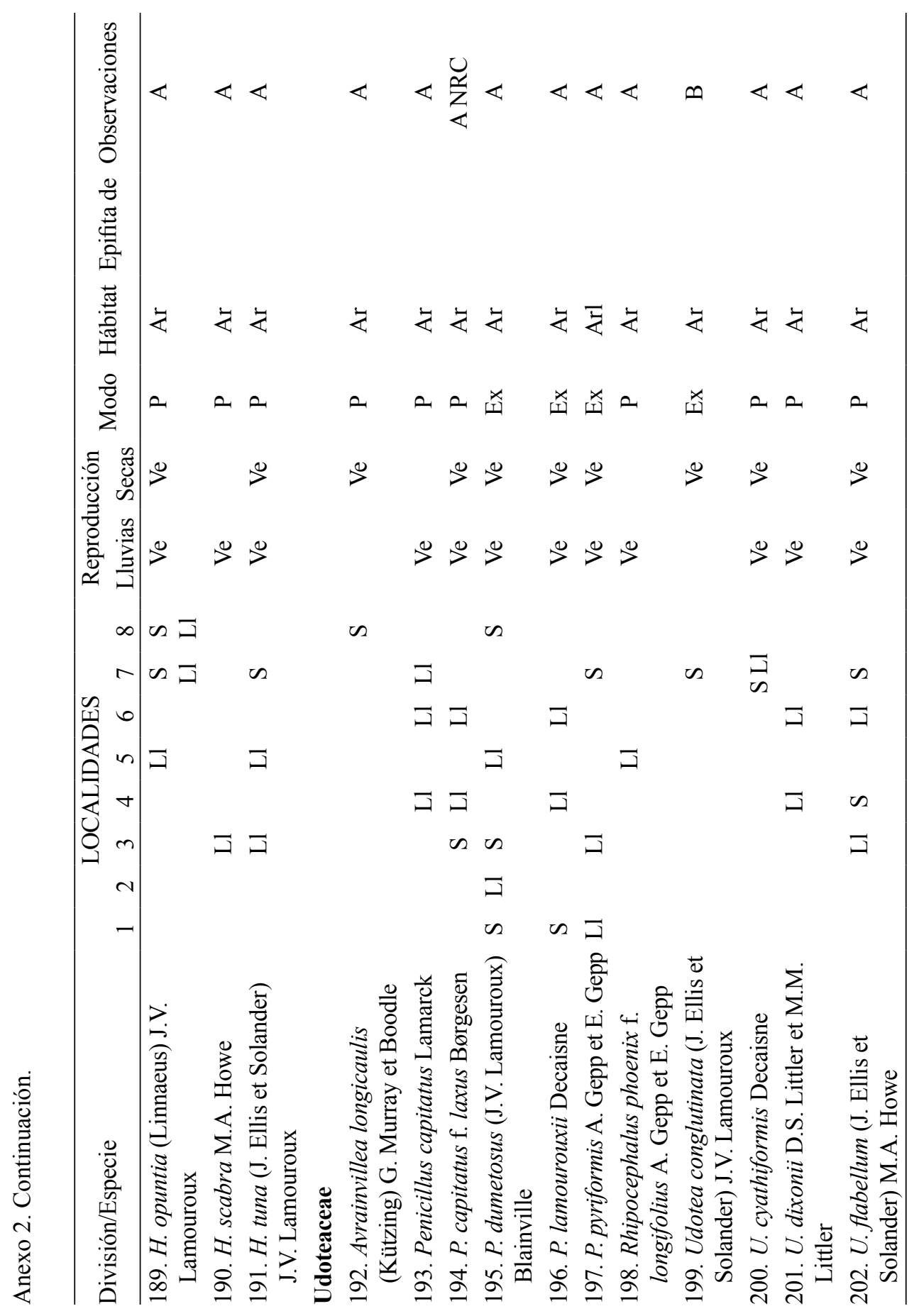

91 
Acta Botanica Mexicana 104: 53-92 (2013)

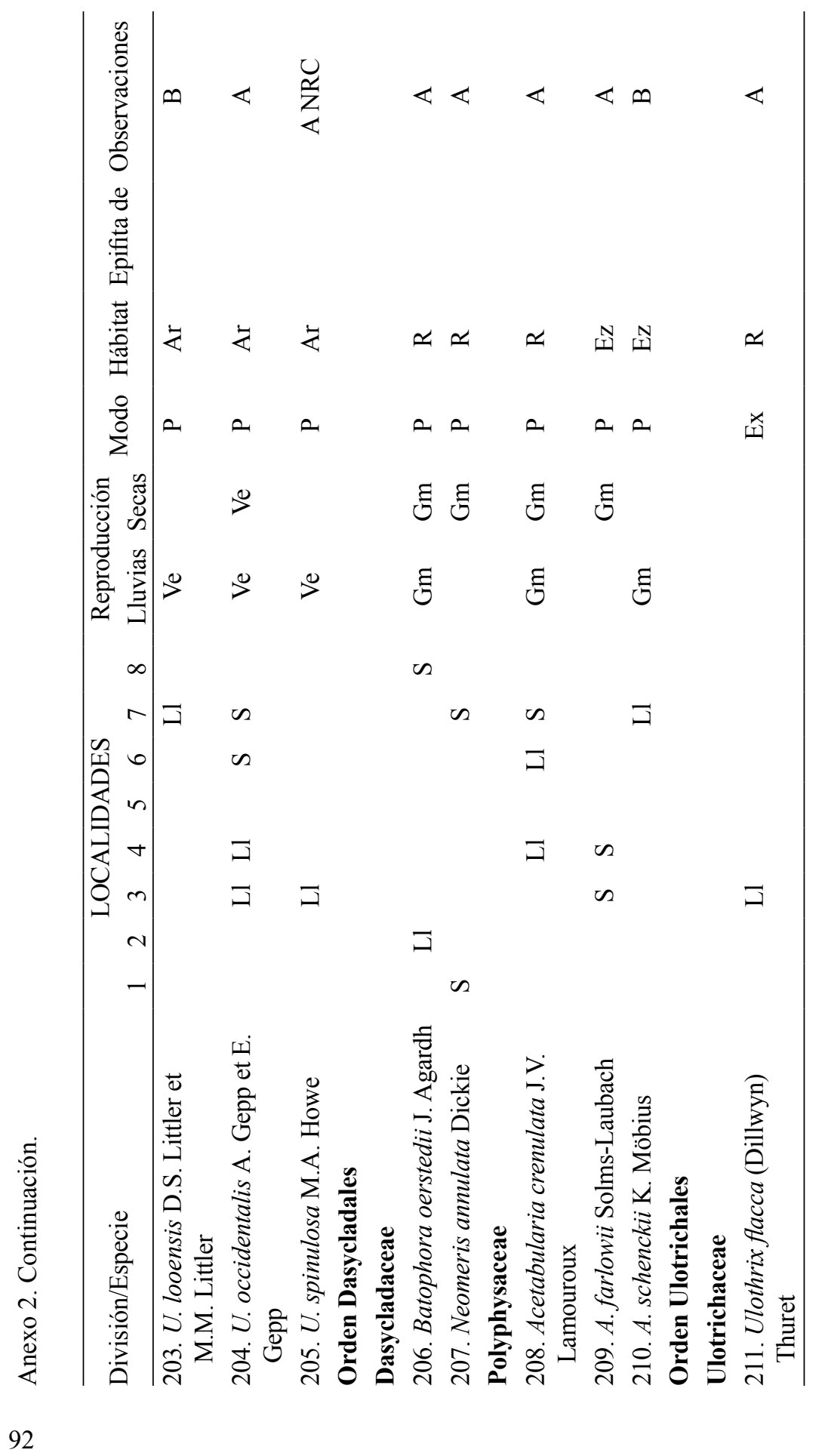

